Check for updates

Cite this: RSC Adv., 2017, 7, 46344

\title{
Some new azobenzene liquid crystals involving chalcone and ester linkages $\dagger$
}

\author{
Xueyou Zhu, Fengnan Yin, Haiying Zhao, (D)* Shufeng Chen (D) and Zhanxi Bian
}

Five new series of azobenzene derivatives containing thiophene, naphthalene or ferrocene with chalcone and ester linkages have been synthesized and characterized. The photosensitive azobenzene group underwent photoisomerization under UV light, which was monitored by UV-visible spectroscopy. The cyclic voltammograms of compounds containing ferrocene showed a quasi-reversible and diffusioncontrolled redox process. These compounds displayed high thermal stability according to the thermogravimetry measurements. According to differential scanning calorimetry, thermal polarizing microscopy and powder X-ray diffraction studies, the compounds containing ferrocene and the compounds with three or no terminal alkoxy chains on the side of the ester group all showed no liquid crystal behaviour. However, the compounds with a terminal alkoxy chain on the side of the ester group or a terminal alkoxy chain at both ends of the molecule exhibited enantiotropic mesophases, but the latter had a narrow mesogenic domain. The increase of the length of the mesogenic unit by replacement of the benzene ring with a naphthalene ring resulted in both an increase in the clearing point and in an increase in the mesophase domain.

Received 22nd June 2017

Accepted 26th September 2017

DOI: $10.1039 / \mathrm{c} 7 \mathrm{ra06958h}$

rsc.li/rsc-advances study the materials in holography, optical storage device, optical switching and establish widespread applications in display technology. ${ }^{14-19}$

On the other hand, chalcones are the main precursors for the biosynthesis of flavonoids and isoflavonoids. A variety of organic molecules with chalcone moiety have been evaluated for pharmacological applications due to their good antimicrobial, ${ }^{20}$ nematocidal, ${ }^{21}$ anticancer ${ }^{22}$ and antiplasmodial ${ }^{23}$ activities. In addition, chalcones are reported to be effective photosensitive materials and have potential nonlinear optical ${ }^{24}$ and liquid crystal properties. ${ }^{25}$ Chalcone linkage along with other linkages, such as imine and ester groups, combined to exhibit various mesophases. ${ }^{26-31}$ However, to the best of our knowledge, there are rare reports about the combination of azo and chalcone linkages using in the liquid-crystalline materials to date..$^{32,33}$ In this article, we synthesized a series of new liquid crystalline compounds involving chalcone, azo and ester linkages. The designed molecular structures are shown in Fig. 1. Their redox and optical properties were studied. Thermometric data will be evaluated in terms of molecular rigidity and flexibility depending on molecular structure and then mesomorphic properties and behaviors will be compared with structurally similar series.

\section{Results and discussion}

\subsection{Photoisomerisation studies}

The photoisomerisation studies were carried out on the selective compounds dissolved in the dichloromethane. The initial 


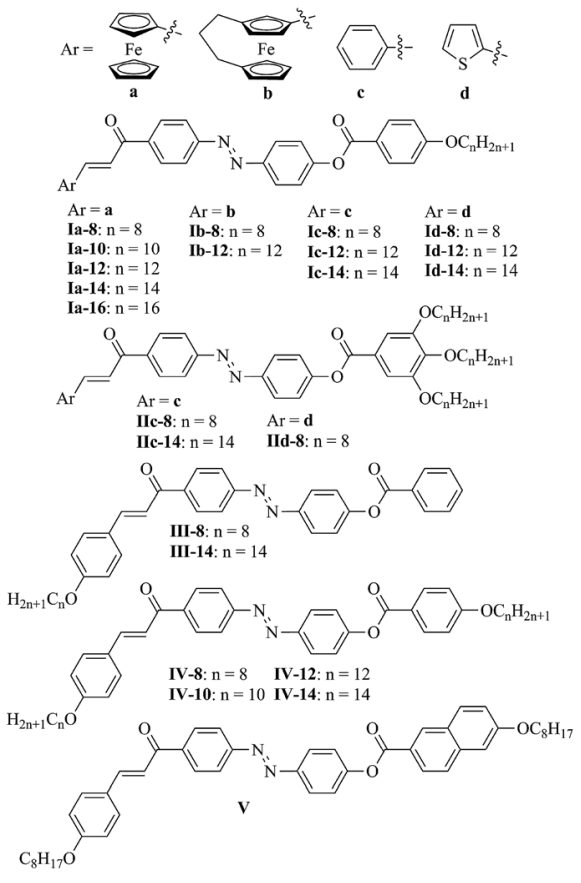

Fig. 1 The structures of compounds I-V.

measurements were carried out on the unexposed materials. The obtained results of the UV-visible absorption spectra (UVvis) are presented in Fig. 2 and Table S1. $\dagger$

Compounds Ia-8 and Ib-8 exhibited three absorption bands, whereas others exhibited two absorption bands. The absorption bands in ultraviolet area were attributed to the $\pi-\pi^{*}$ electronic transitions. For the compound $\mathbf{V}$, the absorption band centered at $\sim 260 \mathrm{~nm}$ (see Fig. 2 insert) had large molar absorption coefficient after introducing the naphthalene ring. The absorption due to $\mathrm{Fe}(\mathrm{d})-\pi^{*}$ electronic transitions in compounds Ia-8 and Ib-8 also appeared at the range of $260 \mathrm{~nm}$. In addition, the same absorption at $346 \mathrm{~nm}$ for compounds Ia, Ib and Ic indicated that the introduction of ferrocenyl almost had no effect on this absorption. However, this band shifted bathochromically about $10 \mathrm{~nm}$ after introduction of thiophene ring (compounds Id and IId) or alkoxy group on the side of chalcone (compounds III, IV and V). Furthermore, compounds Ia-8 and Ib-8 with ferrocenyl showed very broad absorption bands at 480-620 nm due to d-d type transitions of the electrons of the iron atoms, and this band for the [3]ferrocenophane-containing Ib-8 was stronger than that of ferrocene-containing $\mathbf{1 a - 8} .^{34}$

Next, the compounds were irradiated with UV light (365 nm). As an example, the spectral changes of Ic are presented in Fig. 3. With increasing time of irradiation the band at $342 \mathrm{~nm}$ strongly decreased. This change in the UV-vis spectrum suited the reduction of concentration of $E$ isomers in azo group in irradiated mixture. At the same time, the increase of the band at $440 \mathrm{~nm}$ ( $\mathrm{n}-\pi^{*}$ electron transition) corresponded to the growth of the concentration of $Z$ isomer in azo group in investigated mixture. Moreover, the equilibrium between $Z$ and $E$ isomers in azo group was established after $75 \mathrm{~s}$ of irradiation. The isosbestic points were observed at 312 and $408 \mathrm{~nm}$, corresponding

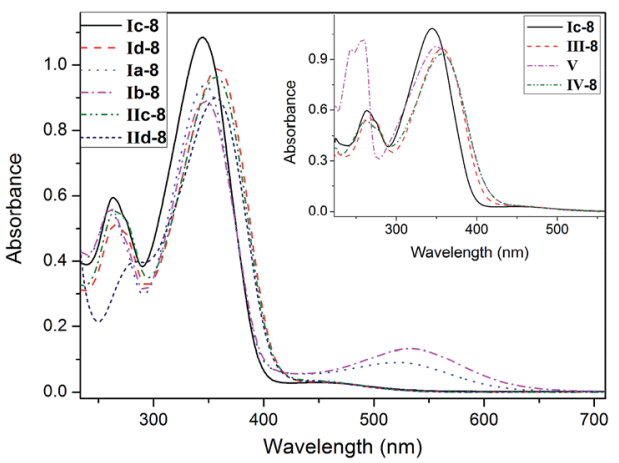

Fig. 2 UV-vis absorption spectra of selective compounds I-V taken at $10^{-5} \mathrm{M}$ in $\mathrm{CH}_{2} \mathrm{Cl}_{2}$.

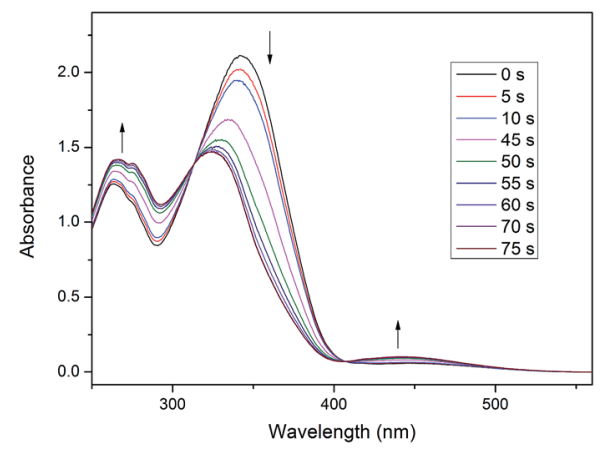

Fig. 3 Changes of absorption spectra of Ic- 8 in $\mathrm{CH}_{2} \mathrm{Cl}_{2}$ during UV exposure.

to $E-Z$ isomerization as these suggesting that only two isomers obtained. Furthermore, another absorption band exhibited chalcone unit in double bond underwent isomerization around $263 \mathrm{~nm} .{ }^{35}$ Table 1 shows the summary of time taken for the selected compounds for $E-Z$ photoisomerisation with their calculated conversion efficiency (CE). ${ }^{7}$ As shown in Table 1, 1a-8 took the longest time (157 s) for $E-Z$ conversion with the CE of $27.3 \%$ after exposure to UV light, whereas Ic- 8 gave $45.1 \%$ of CE when bulky ferrocene was replaced by a benzene ring. However, Id-8, IV-8 and V-8 took shorter time (40-50 s) with the low CE of $8-10 \%$ after introducing alkoxy chain in benzene ring. The possible reason is the bulky ferrocene and alkoxy chain increasing the energy of $Z$ isomer.

After irradiation samples were left in the dark to allow thermal back relaxation. This process for Ic-8 is presented in Fig. 4. It can be seen that back transition from $Z$ to $E$ form was much slower than the isomerisation during the UV irradiation, and the equilibrium for compound Ic-8 was not achieved yet after $1485 \mathrm{~min}$. Similar phenomena were observed in other compounds. Long thermal back relaxation allows us to realize that optical storage devices with these materials which need longer periods.

\subsection{Electrochemical investigation}

The electrochemical properties of representative compounds containing ferrocene (Ia-8, Ia-12 and Ib-12) were studied by 
Table 1 Time taken for the azo derivatives for $E-Z$ isomerisation with their calculated conversion efficiency

\begin{tabular}{lcr}
\hline Compd. & $E-Z($ time $)(\mathrm{s})$ & $\mathrm{CE}(\%)$ \\
\hline Ia-8 & 157 & 27.3 \\
Ic-8 & 75 & 45.1 \\
Id-8 & 40 & 9.6 \\
IV-8 & 50 & 10.2 \\
V-8 & 40 & 8.7
\end{tabular}

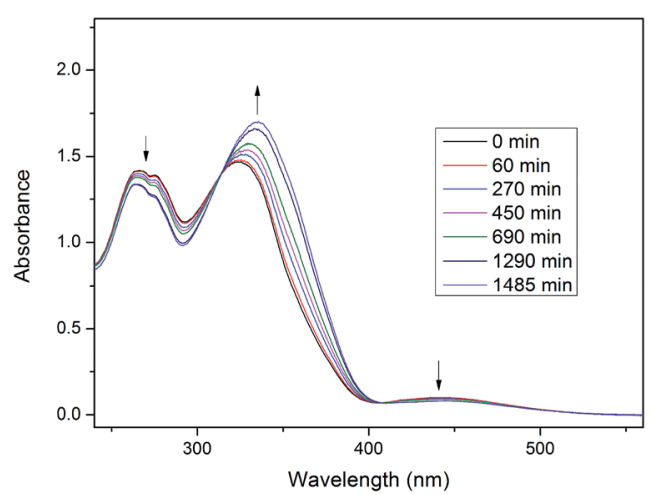

Fig. 4 Changes of absorption spectra of Ic- 8 in $\mathrm{CH}_{2} \mathrm{Cl}_{2}$ during thermal back relaxation.

cyclic voltammetry (CV) using $\mathrm{CH}_{2} \mathrm{Cl}_{2}$ as the solvent containing $0.1 \mathrm{M}$ tetra- $n$-butylammoniumhexafluorophosphate $\left(\mathrm{TBAPF}_{6}\right)$ as a supporting electrolyte. Electrochemical data are shown in Table 2. Selected CV curves are shown in Fig. 5. All redox-active ferrocenyl groups exhibited one-electron transfer processes with formal redox potentials $E_{1 / 2}=146-148 \mathrm{mV}$ for Ia-8, Ia-12 and $E_{1 / 2}=79 \mathrm{mV}$ for $\mathbf{I b}-\mathbf{1 2}$ vs. $\mathrm{Fc} / \mathrm{Fc}^{+}$, which indicates that lengthening of the alkoxy chain has little effect on the formal redox potentials of ferrocene. However, as compared to Ia-8 and Ia-12, the potentials for Ib-12 cathodically shifted about $70 \mathrm{mV}$ thereby suggesting easy oxidation by loss of an electron for [3] ferrocenophane-containing derivatives. A reasonable explanation is the effect of trimethylene group as an electron donor in [3]ferrocenophane-containing derivative leading to the lower oxidation potential of ferrocene. ${ }^{36}$ From Table 2, we found the $i_{\mathrm{pc}} / i_{\mathrm{pa}}$ ratios approached 1 , but the $\Delta E$ value was in the range of 143-149 mV indicating a quasi-reversible redox step for studied

Table $2 \mathrm{CV}$ data of $10^{-3} \mathrm{M}$ solutions of selected compounds in $\mathrm{CH}_{2} \mathrm{Cl}_{2}$ containing $0.1 \mathrm{M} \mathrm{TBAPF}_{6}$ as supporting electrolyte at $100 \mathrm{mV}$ $\mathrm{s}^{-1}$ scan rate. Potentials are vs. Fc/Fc ${ }^{+}$

\begin{tabular}{lllllll}
\hline Compd. & $E_{\mathrm{pa}}{ }^{a} / \mathrm{mV}$ & $E_{\mathrm{pc}}{ }^{b} / \mathrm{mV}$ & $\Delta E^{c} / \mathrm{mV}$ & $E_{1 / 2}{ }^{d} / \mathrm{mV}$ & $i_{\mathrm{pa}}{ }^{e} / \mu \mathrm{A}$ & $i_{\mathrm{pa}} / i_{\mathrm{pc}}{ }^{f}$ \\
\hline Ia-8 & 223 & 74 & 149 & 148 & 27.4 & 0.96 \\
Ia-12 & 217 & 74 & 143 & 146 & 25.0 & 0.89 \\
Ib-12 & 151 & 6 & 145 & 79 & 29.4 & 0.99
\end{tabular}

${ }^{a}$ Anodic peak potential. ${ }^{b}$ Cathodic peak potential. ${ }^{c} \Delta E=E_{\mathrm{pa}}-E_{\mathrm{pc} \cdot}$. ${ }^{d} E_{1 / 2}=1 / 2\left(E_{\mathrm{pa}}+E_{\mathrm{pc}}\right) .{ }^{e}$ Anodic peak current. ${ }^{f} i_{\mathrm{pc}}$, cathodic peak current.

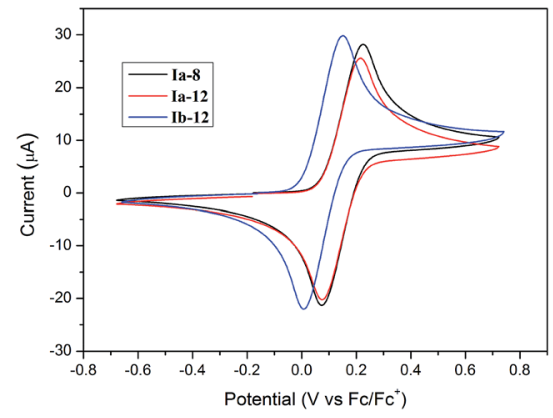

Fig. $5 \mathrm{CV}$ curves of selective compounds la-lb in $\mathrm{CH}_{2} \mathrm{Cl}_{2}, 0.1 \mathrm{M}$ TBAPF $_{6}$. Scan rate $=100 \mathrm{mV} \mathrm{s}^{-1}$.

compounds. In addition, as shown in Fig. 6, the redox potentials of Ib-8 slightly influenced by the scan rate in a range from 10 to $300 \mathrm{mV} \mathrm{s}^{-1}$, and both the anodic and cathodic peak currents are linear to the square root of scan rates, indicating a diffusion-controlled process. ${ }^{34}$

\subsection{Thermal behavior}

Thermal stability of these compounds was measured by simultaneous thermogravimetric and differential scanning calorimetry (TG-DSC) measurement under $\mathrm{N}_{2}$. The TG curves of Ia-Id are shown in Fig. S1. $\dagger$ From the TG curves, we can see that the decomposition temperatures were somewhat higher for Ic-8 and Id-8 (about $300{ }^{\circ} \mathrm{C}$ ) than for Ia-8 and Ib-8 (about $270{ }^{\circ} \mathrm{C}$ ), which indicated the higher thermal stability of the former.

Mesomorphic properties of these compounds had been investigated. The textural studies of them have been performed using thermal polarizing microscopy (POM) and the thermal analyses have been carried out using DSC. The thermal analyses data of compounds Ia, Ib, II and III are presented in Table S2. $\dagger$ As shown in Table $\mathrm{S} 2, \uparrow$ compounds Ia-Ib did not display LC behaviors, however, most of them gave rise to crystal to crystal or crystal polymorphic phase transitions upon heating and cooling cycles. For the series of compounds Ia, the phase transition temperature of crystal to isotropic liquid lowered gradually with increasing the length of the terminal alkoxy chain from eight to fourteen carbon atoms, but this temperature raised when further lengthening the terminal alkoxy chain to sixteen

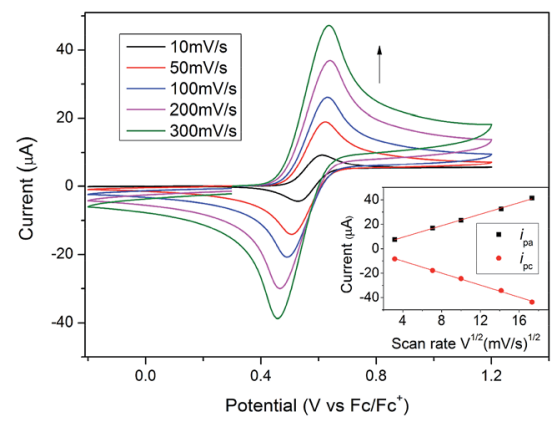

Fig. $6 \mathrm{CV}$ curves and peak currents of $\mathrm{Ib}-8$ obtained at different scan rates in $\mathrm{CH}_{2} \mathrm{Cl}_{2}$. 
carbon atoms. In addition, this temperature for the series of compounds Ib with the same length of terminal alkoxy chain lowered obviously when replacing ferrocene by [3] ferrocenophane.

Compounds Ia-Ib were non-mesomorphic. It seems to like that there is unfavourable effect of bulky ferrocene in present system which is disadvantageous to molecule packing, so ferrocene was replaced by a benzene ring or thiophene ring in the following study. Compounds Ic-Id with one terminal alkoxy chain on the side of ester group were prepared, and we were pleased to find that they all displayed liquid crystal behaviors and showed similar phase transitions in the heating and cooling cycles. As examples, the DSC curves of Ic-8 and Id-8 are shown in Fig. 7. At the beginning of the first heating cycle, crystal to crystal phase transition was observed for Ic-8, then an endothermic peak appeared at $167{ }^{\circ} \mathrm{C}$ with large enthalpy change of $82.0 \mathrm{~kJ} \mathrm{~mol}^{-1}$ corresponding to the melting point followed by the formation of mesophase with the range of $61{ }^{\circ} \mathrm{C}$. The mesophase exhibited marbled texture as presented in Fig. 8a which was identified by POM..$^{37}$ On slow cooling from the isotropic liquid, the enantiotropic mesophase was observed. The droplet texture for Ic-8, fourbrush schlieren texture for Ic-12 and marbled texture for Ic-14 appeared on cooling as presented in Fig. 8b-d. X-ray diffraction (XRD) experiments were further performed to elucidate the mesomorphic properties. As shown in Fig. 9, the XRD patterns of Ic-8 presented one diffuse peak in wide-angle region during the heating and cooling cycle after entering into the mesophase, which demonstrated a disordered position of mesogen. Taking into account the DSC, POM and XRD results, this series compounds probably possessed enantiotropic smectic C (SmC) phase. ${ }^{38}$ As shown in Table 3, the mesophase ranges $(\Delta T)$ in the cooling cycles were $79.1^{\circ} \mathrm{C}$ for Ic8, $56.4{ }^{\circ} \mathrm{C}$ for Ic-12 and $51.5{ }^{\circ} \mathrm{C}$ for Ic-14 respectively, which narrowed gradually with increasing the length of the terminal alkoxy chain. The lower homologues Ic-8 had the widest mesogenic domain and the highest clearing temperature $\left(\sim 229^{\circ} \mathrm{C}\right)$. Similar thermal behaviors were observed in series Id. Moreover, for the compound with same length of terminal alkoxy chain, the mesophase range of the series Id containing thiophene $\left(91.0{ }^{\circ} \mathrm{C}\right.$ for Id-8, $66.0^{\circ} \mathrm{C}$ for Id-12 and $58.7^{\circ} \mathrm{C}$ for Id14) was wider, and the clearing points of series Id were higher

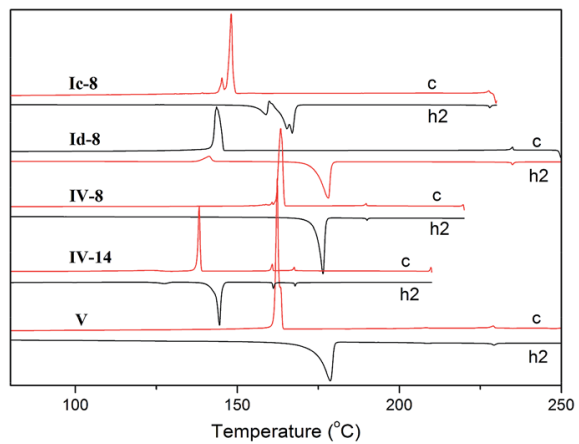

Fig. 7 DSC traces of selected compounds. h2: the second heating, c: the first cooling.

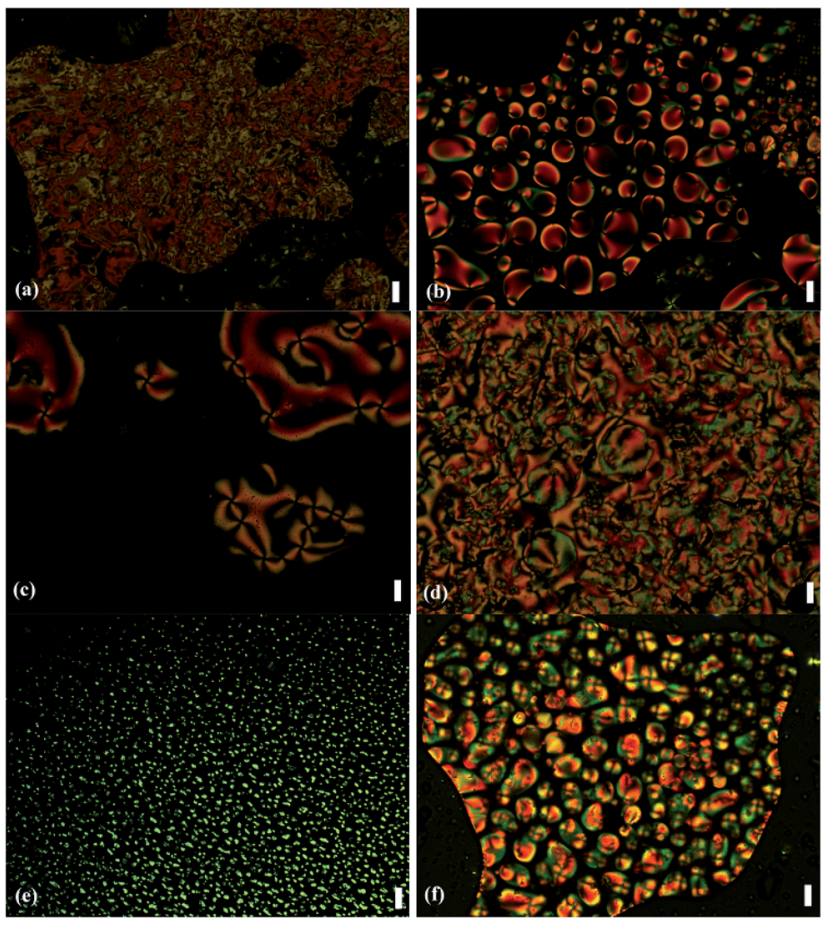

Fig. 8 Polarizing optical microscopy images $(20 \times)$ (a) Ic -8 at $195.0^{\circ} \mathrm{C}$ on heating, (b) Ic-8 at $229.8^{\circ} \mathrm{C}$ (c) Ic-12 at $207.4{ }^{\circ} \mathrm{C}$ (d) Ic-14 at $200.4{ }^{\circ} \mathrm{C}$ (e) Id -8 at $232.6^{\circ} \mathrm{C}$ (f) Id -12 at $213.0^{\circ} \mathrm{C}$ on cooling. Scale bar, $30 \mu \mathrm{m}$.

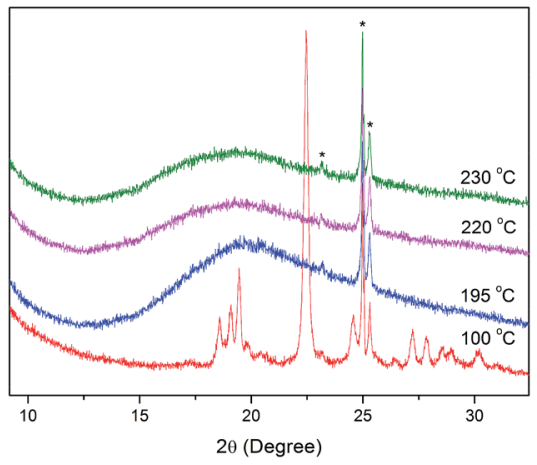

Fig. 9 XRD patterns of compound Ic- 8 on heating $195^{\circ} \mathrm{C}$ and cooling $230^{\circ} \mathrm{C}, 220^{\circ} \mathrm{C}$ and $100^{\circ} \mathrm{C}$. Asterisks in the spectrum show the alumina from the sample cell holder.

than those of the series Ic. The POM images of Id-8 at $232.6{ }^{\circ} \mathrm{C}$ and Id-12 at $213.0^{\circ} \mathrm{C}$ on cooling were presented in Fig. 8e and f. Therefore, the presence of heteroatom $\mathrm{S}$ being more polarizable than carbon resulted in great changes in the corresponding liquid crystalline phases and physical properties of the observed phases.

Compounds Ic-Id all exhibited enantiotropic mesomorphic behaviours, but their melting points were high. In order to obtain mesogenic compounds with low melting point, compounds IIc-IId with three terminal alkoxy chains were prepared. As shown in Table $\mathrm{S} 2, \uparrow$ the melting points of compounds IIc-8, IIc-14 and IId-8 are $73.5,72.7$, and $94.1{ }^{\circ} \mathrm{C}$ 
Table 3 Phase transition temperatures and associated enthalpies of compounds 1-4

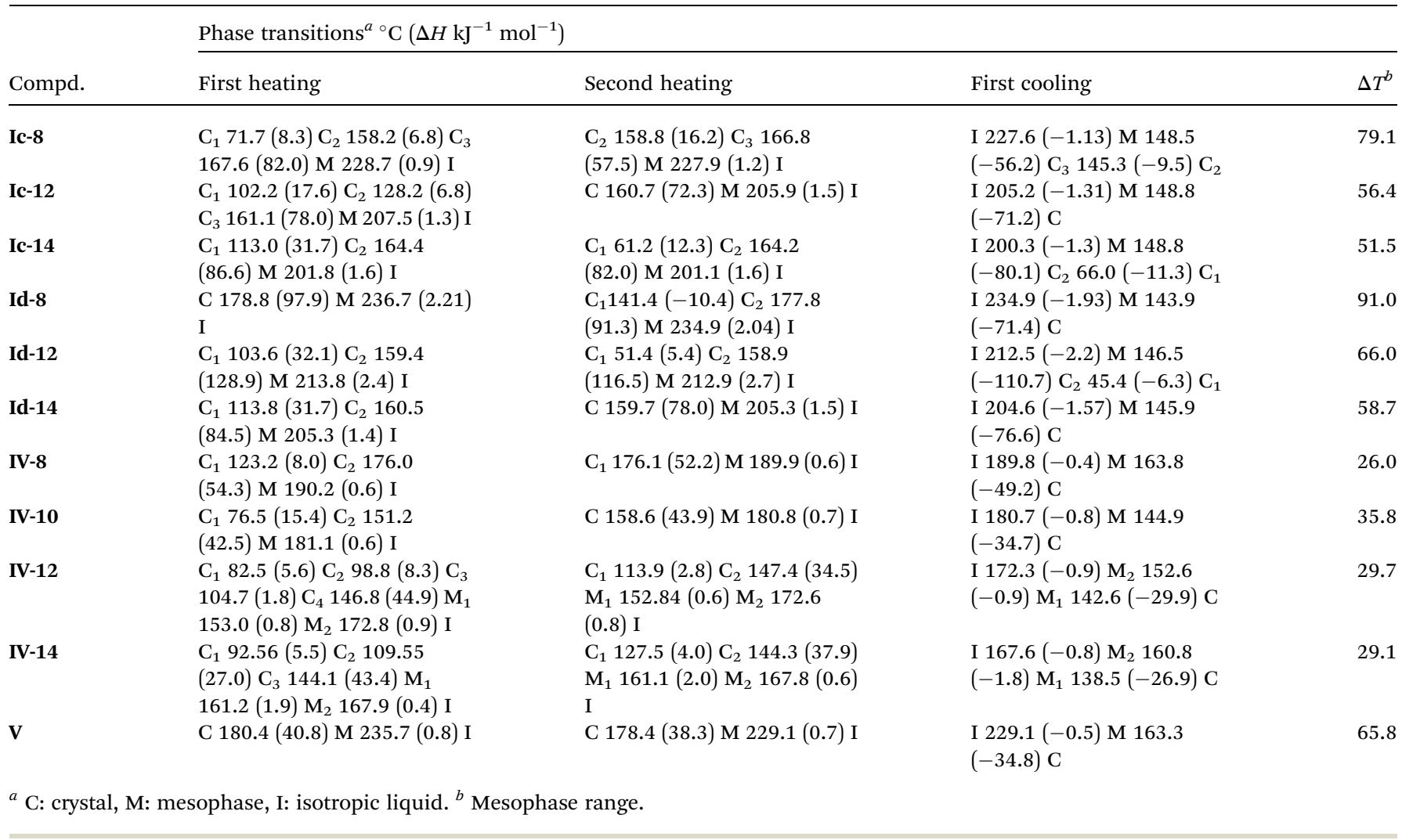

respectively, lowering $85-94{ }^{\circ} \mathrm{C}$ compared with compounds Ic-8, Ic-14 and Id-8. However, compounds IIc-IId with three terminal alkoxy chains were non-mesomorphic, but showed either simple melting and freezing process or crystal polymorphic phase transitions in the heating and cooling cycles. From above observations, it seemed likely that increasing the number of long chain was unfavourable for inducing mesomorphism due to increasing the disorder of molecular.
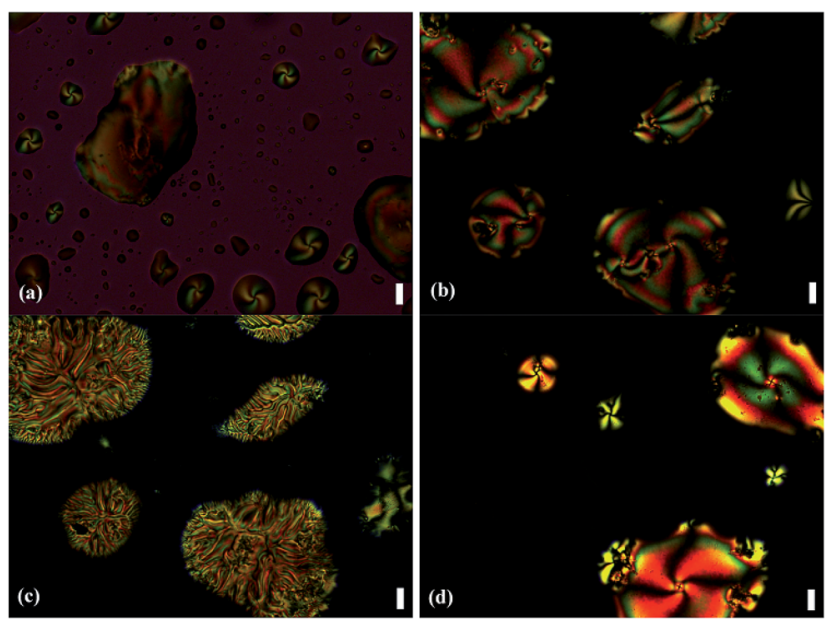

Fig. 10 Polarizing optical microscopy images $(20 \times)$ (a) IV-8 at $192.4^{\circ} \mathrm{C}$ on heating, (b) IV-14 at $169.6^{\circ} \mathrm{C}$ (c) IV-14 at $162.7^{\circ} \mathrm{C}$ (d) V at $239.6^{\circ} \mathrm{C}$ on cooling. Scale bar, $30 \mu \mathrm{m}$.
To better understand the effect of the position of long chain on the formation of mesophases, compounds III-8 and III-14 with terminal alkoxy chain on the side of chalcone were prepared. We were surprised to find that compounds III-8 and III-14 showed no liquid crystal behaviours. As shown in Table S2, $\dagger$ compounds III-8 and III-14 also displayed crystal polymorphic phase transitions in the heating and cooling cycles, and their melting points (III-8, $184.8{ }^{\circ} \mathrm{C}$; III-14, $173.6{ }^{\circ} \mathrm{C}$ ) were slight higher than those of compounds Ic-8 $\left(167.6^{\circ} \mathrm{C}\right)$ and Ic-14 $\left(164.4^{\circ} \mathrm{C}\right)$.

Next, we studied the thermal behaviours of compounds IV-V with a terminal alkoxy chain at both ends of the molecular. From Table 3, we can see that the melting point of compound IV-8 $\left(176.0{ }^{\circ} \mathrm{C}\right)$ increased by about $10{ }^{\circ} \mathrm{C}$ compared with $1 \mathrm{c}-8$ $\left(167.6^{\circ} \mathrm{C}\right)$, whereas the increase of the length of the flexible chain $\left(\mathbf{I V}-12,146.8^{\circ} \mathrm{C}\right.$; IV-14, $\left.144.1^{\circ} \mathrm{C}\right)$ resulted in a decrease of the melting point by $15-20{ }^{\circ} \mathrm{C}$ compared with Ic-12 $\left(161.1^{\circ} \mathrm{C}\right)$ and Ic-14 (164.4 $\left.{ }^{\circ} \mathrm{C}\right)$. According DSC and POM observation, the series IV all showed enantiotropic mesophases but with the narrow mesogenic domains $\left(26-36{ }^{\circ} \mathrm{C}\right)$ and low clearing temperature compared with the series of Ic. Upon heating, compounds IV-8 and IV-10 having shorter chains exhibited schlieren texture after entering into the mesophase, and showed fourfold brush near the melting point (Fig. 10a). The inversing process was also observed on cooling. However, as shown in Table 3, IV-12 and IV-14 having longer chains displayed two mesogenic domains. As an example, on cooling from isotropic of IV-14, fourbrush schlieren texture was observed at 


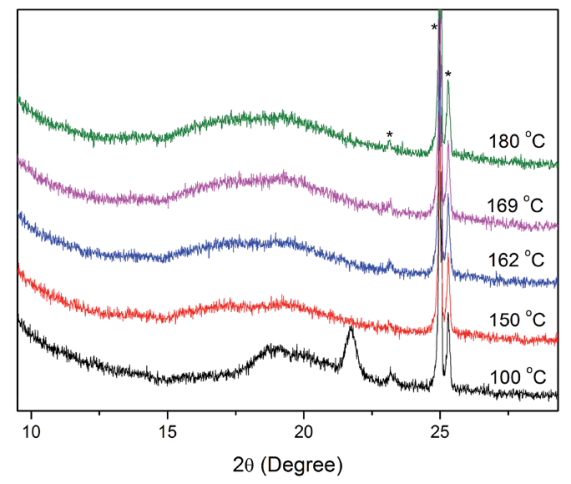

Fig. 11 XRD patterns of compound IV-14 on cooling. Asterisks in the spectrum show the alumina from the sample cell holder.

$169.6^{\circ} \mathrm{C}$ as displayed in Fig. 10b and further cooling, dendritic texture were exhibited at $162.7^{\circ} \mathrm{C}$ as displayed in Fig. 10c. ${ }^{39}$ The XRD patterns of IV-14 (Fig. 11) presented one diffuse peak during the cooling cycle after entering into the mesophase indicating the possibility of SmC phase.

The increase of the length of the mesogenic unit by replacement benzene ring with naphthalene ring (compound $\mathbf{V}$ ) resulted in both an increase of the clearing point by about $46^{\circ} \mathrm{C}$ compared with its analogue, compound IV-8, and in an increase of the SmC mesophase domain (by about $40^{\circ} \mathrm{C}$ ), but the melting point changed slightly. Fig. 10d showed the POM image of $\mathbf{V}$ at $239.6{ }^{\circ} \mathrm{C}$ on cooling. Thus, the lengthening the rigid core may favour a better packing of the molecules which was beneficial to exhibiting liquid crystal properties.

\section{Conclusion}

In summary, we have synthesized 23 azobenzene compounds involving chalcone and ester linkages. The compounds containing ferrocene exhibit good electrochemical properties. Investigations of liquid crystal properties indicated that the compounds containing ferrocene (Ia-Ib series) and the compounds with three (IIc-IId series) or no (III series) terminal alkoxy chains on the side of ester group all showed no liquid crystal behaviours. However, the compounds with a terminal alkoxy chain on the side of ester group (Ic-Id series) or a terminal alkoxy chain at both ends of the molecular (IV-V series) exhibited enantiotropic SmC phases. The replacement benzene ring with naphthalene ring results in both an increase of the clearing point and in an increase of the mesophase domain. Some of these azo molecules underwent photoisomerization under UV light. Very long thermal back relaxation has potential advantage in the creation of optical storage devices.

\section{Experimental}

\subsection{Materials and measurements}

Detailed procedures of synthesis and characterization of compounds VIa-VIe were described in ESI. $\dagger$ Acyl chloride VII was prepared according to reported procedures. ${ }^{25}$ IR spectra were recorded as $\mathrm{KBr}$ pellets on a Bruker-ALPHA spectrometer.
NMR spectra were recorded on an Avance 500 Bruker (500 MHz) spectrometer using tetramethylsilane as internal standard. HRMS spectra were recorded on a Bruker ultrafleXtreme MALDITOF/TOF mass spectrometer. Elemental analysis was performed on a Perkins-Elmer 2400 elemental analyser. Electronic absorption spectra were recorded on Shimadzu UV2600 spectrophotometer. Thermal stability was measured by EXSTAR TG/ DTA7300 simultaneous TG-DSC measurement under $\mathrm{N}_{2}$. DSC thermographs were obtained on a METTLER TOLEDO DSC3 at a heating rate of $10^{\circ} \mathrm{C} \mathrm{min}^{-1}$ under nitrogen flow. Powder XRD experiments were conducted using a D8 ADVANCE equipped with a LynxEye detector, $\mathrm{CuK} \alpha$ radiation source, and graphite monochromator, $\lambda=1.54 \AA$. CV experiments were recorded on AUTOLAB PGSTAT302 voltammetric analyzer and performed at room temperature in dry $\mathrm{CH}_{2} \mathrm{Cl}_{2}$ solutions with the concentration of $10^{-3} \mathrm{M}$ containing $0.1 \mathrm{M} \mathrm{TBAPF}_{6}$ as a supporting electrolyte. A three-electrode configuration consisting of a glassy carbon working electrode, a Pt wire counter electrode, and an Ag/ $\mathrm{AgCl}$ (with a saturated $\mathrm{KCl}$ solution) couple reference electrode was used. The $\mathrm{Fc} / \mathrm{Fc}^{+}$couple was used as internal reference and showed a peak at $+0.484 \mathrm{~V} v$ s. Ag/ $\mathrm{AgCl}$. Prior to experiments, the system was purged with purified nitrogen gas to exclude dissolved oxygen from the solution.

\subsection{General procedure for the synthesis of compounds I-V}

Compound VI $(0.45 \mathrm{mmol})$ was redissolved in dry pyridine $(2 \mathrm{~mL})$, to which acyl chloride VII $(0.5 \mathrm{mmol})$ in dry pyridine $(2 \mathrm{~mL})$ was added dropwise at $-5{ }^{\circ} \mathrm{C}$. The reaction was stirred for $4 \mathrm{~h}$ at room temperature. The solvent was removed by distillation at reduced pressure and the residue was separated by silica gel column chromatography using $\mathrm{CH}_{2} \mathrm{Cl}_{2} /$ petroleum ether as eluent. The second fraction was the desired product, which was recrystallized from $\mathrm{CH}_{2} \mathrm{Cl}_{2} /$ methanol to afford the pure compounds I-V (Scheme 1).

Ia-8. Yield $67 \%, \mathrm{mp} 172-178{ }^{\circ} \mathrm{C}, R_{\mathrm{f}}=0.117\left(\mathrm{CH}_{2} \mathrm{Cl}_{2}\right.$ : petroleum ether $=2: 1)$; $\mathrm{IR}\left(\mathrm{KBr}, \nu, \mathrm{cm}^{-1}\right): 3102,2950,2919,2853,1733$, 1654, 1592, 1509, 1315, 1261, 1074, 979; ${ }^{1} \mathrm{H}$ NMR $(500 \mathrm{MHz}$, $\left.\mathrm{CDCl}_{3}\right)(\delta \mathrm{ppm}): 8.17-8.13\left(\mathrm{~m}, 4 \mathrm{H}, \mathrm{C}_{6} \mathrm{H}_{4}\right), 8.06-8.00\left(\mathrm{~m}, 4 \mathrm{H}, \mathrm{C}_{6} \mathrm{H}_{4}\right)$, $7.81(\mathrm{~d}, J=15.0 \mathrm{~Hz}, 1 \mathrm{H}, \mathrm{CH}), 7.41\left(\mathrm{~d}, J=8.5 \mathrm{~Hz}, 2 \mathrm{H}, \mathrm{C}_{6} \mathrm{H}_{4}\right), 7.17(\mathrm{~d}$, $J=15.0 \mathrm{~Hz}, 1 \mathrm{H}, \mathrm{CH}), 7.00\left(\mathrm{~d}, J=9.0 \mathrm{~Hz}, 2 \mathrm{H}, \mathrm{C}_{6} \mathrm{H}_{4}\right), 4.63(\mathrm{~d}, J=$ $2.0 \mathrm{~Hz}, 2 \mathrm{H}, \mathrm{FcH}), 4.52$ (d, $J=2.0 \mathrm{~Hz}, 2 \mathrm{H}, \mathrm{FcH}), 4.21(\mathrm{~s}, 5 \mathrm{H}, \mathrm{FcH})$,
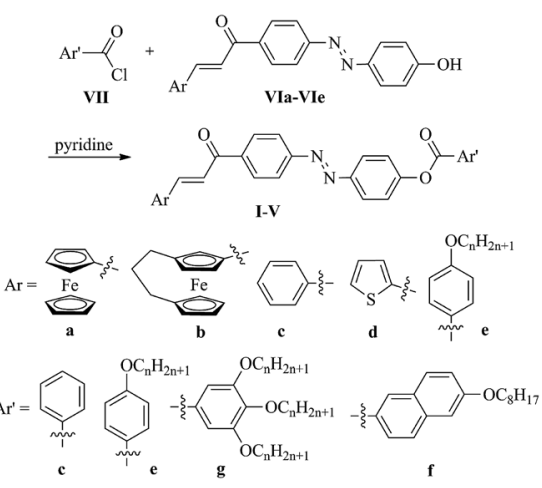

Scheme 1 Synthesis of compounds I-V. 
$4.06\left(\mathrm{t}, J=6.5 \mathrm{~Hz}, 2 \mathrm{H}, \mathrm{OCH}_{2}\right), 1.88-1.78\left(\mathrm{~m}, 2 \mathrm{H}, \mathrm{CH}_{2}\right), 1.52-1.44$ (m, 2H, $\mathrm{CH}_{2}$ ), 1.37-1.45 (m, 8H, $\left.\mathrm{CH}_{2}\right), 0.90\left(\mathrm{t}, J=7.0 \mathrm{~Hz}, 3 \mathrm{H}, \mathrm{CH}_{3}\right)$; ${ }^{13} \mathrm{C}$ NMR (125 MHz, $\mathrm{CDCl}_{3}$ ) ( $\left.\delta \mathrm{ppm}\right): 188.97,164.62,163.82$, $154.62,153.76,150.23,147.55,132.45,129.49,124.49,122.98$, 122.65, 121.17, 114.46, 72.74, 72.30, 70.58, 69.67, 68.44, 31.86, 29.38, 29.28, 26.04, 22.71, 14.16; HRMS, MALDI ${ }^{+}, \mathrm{m} / \mathrm{z}$ : calcd for $\mathrm{C}_{40} \mathrm{H}_{40} \mathrm{FeN}_{2} \mathrm{O}_{4}:\left[\mathrm{M}^{+}\right]$668.2337, found: 668.2333.

Ia-10. Yield $71 \%$, mp $182-184{ }^{\circ} \mathrm{C}, R_{\mathrm{f}}=0.263\left(\mathrm{CH}_{2} \mathrm{Cl}_{2}\right.$ : petroleum ether $=3: 1)$; IR $\left(\mathrm{KBr}, \nu, \mathrm{cm}^{-1}\right): 3051,2922,2851,1731$, 1654, 1590, 1512, 1316, 1267, 1104, 978; ${ }^{1} \mathrm{H}$ NMR $(500 \mathrm{MHz}$, $\left.\mathrm{CDCl}_{3}\right)(\delta \mathrm{ppm}): 8.16\left(\mathrm{~d}, J=8.5 \mathrm{~Hz}, 2 \mathrm{H}, \mathrm{C}_{6} \mathrm{H}_{4}\right), 8.12(\mathrm{~d}, J=7.0 \mathrm{~Hz}$, $\left.2 \mathrm{H}, \mathrm{C}_{6} \mathrm{H}_{4}\right), 8.05\left(\mathrm{~d}, J=8.5 \mathrm{~Hz}, 2 \mathrm{H}, \mathrm{C}_{6} \mathrm{H}_{4}\right), 8.01(\mathrm{~d}, J=8.0 \mathrm{~Hz}, 2 \mathrm{H}$, $\left.\mathrm{C}_{6} \mathrm{H}_{4}\right), 7.75(\mathrm{~d}, J=14.0 \mathrm{~Hz}, 1 \mathrm{H}, \mathrm{CH}), 7.40(\mathrm{~d}, J=9.0 \mathrm{~Hz}, 2 \mathrm{H}$, $\left.\mathrm{C}_{6} \mathrm{H}_{4}\right), 7.13(\mathrm{~d}, J=14 \mathrm{~Hz}, 1 \mathrm{H}, \mathrm{CH}), 6.99\left(\mathrm{~d}, J=9.0 \mathrm{~Hz}, 2 \mathrm{H}, \mathrm{C}_{6} \mathrm{H}_{4}\right)$, $4.76(\mathrm{~s}, 2 \mathrm{H}, \mathrm{FcH}), 4.64(\mathrm{~s}, 2 \mathrm{H}, \mathrm{FcH}), 4.33(\mathrm{~s}, 5 \mathrm{H}, \mathrm{FcH}), 4.06(\mathrm{t}, J=$ $\left.6.5 \mathrm{~Hz}, 2 \mathrm{H}, \mathrm{OCH}_{2}\right), 1.86-1.80\left(\mathrm{~m}, 2 \mathrm{H}, \mathrm{CH}_{2}\right), 1.51-1.45(\mathrm{~m}, 2 \mathrm{H}$, $\left.\mathrm{CH}_{2}\right), 1.41-1.19\left(\mathrm{~m}, 12 \mathrm{H}, \mathrm{CH}_{2}\right), 0.89\left(\mathrm{t}, J=7.0 \mathrm{~Hz}, 3 \mathrm{H}, \mathrm{CH}_{3}\right) ;{ }^{13} \mathrm{C}$ NMR (125 MHz, $\mathrm{CDCl}_{3}$ ) ( $\left.\delta \mathrm{ppm}\right): 189.09,164.59,163.78,154.58$, 153.72 , 150.19, 147.54, 132.41, 129.39, 124.45, 122.93, 122.61, 121.13, 119.02, 114.42, 71.61, 69.90, 69.16, 68.40, 31.91, 29.57, 29.36, 29.11, 26.00, 22.70, 14.14; HRMS, MALDI ${ }^{+}, \mathrm{m} / \mathrm{z}$ : calcd for $\mathrm{C}_{42} \mathrm{H}_{44} \mathrm{FeN}_{2} \mathrm{O}_{4}:\left[\mathrm{M}^{+}\right]$696.2651, found: 696.2648.

Ia-12. Yield $66.5 \%, \mathrm{mp} 164-168{ }^{\circ} \mathrm{C}, R_{\mathrm{f}}=0.27\left(\mathrm{CH}_{2} \mathrm{Cl}_{2}\right.$ : petroleum ether $=3: 1)$; IR $\left(\mathrm{KBr}, \nu, \mathrm{cm}^{-1}\right): 3088,2920,2850,1728$, 1653, 1602, 1510, 1314, 1256, 1070, 979; ${ }^{1} \mathrm{H}$ NMR $(500 \mathrm{MHz}$, $\left.\mathrm{CDCl}_{3}\right)(\delta \mathrm{ppm}): 8.16\left(\mathrm{~d}, J=8.5 \mathrm{~Hz}, 2 \mathrm{H}, \mathrm{C}_{6} \mathrm{H}_{4}\right), 8.12(\mathrm{~d}, J=8.0 \mathrm{~Hz}$, $\left.2 \mathrm{H}, \mathrm{C}_{6} \mathrm{H}_{4}\right), 8.05\left(\mathrm{~d}, J=8.5 \mathrm{~Hz}, 2 \mathrm{H}, \mathrm{C}_{6} \mathrm{H}_{4}\right), 8.01\left(\mathrm{~s}, 2 \mathrm{H}, \mathrm{C}_{6} \mathrm{H}_{4}\right), 7.73$ (b, 1H, CH), 7.40 (d, J=8.5 Hz, 2H, $\left.\mathrm{C}_{6} \mathrm{H}_{4}\right), 7.14$ (b, 1H, CH), 6.99 $\left(\mathrm{d}, J=8.5 \mathrm{~Hz}, 2 \mathrm{H}, \mathrm{C}_{6} \mathrm{H}_{4}\right), 4.83(\mathrm{~s}, 2 \mathrm{H}, \mathrm{FcH}), 4.69(\mathrm{~s}, 2 \mathrm{H}, \mathrm{FcH})$, $4.37(\mathrm{~s}, 5 \mathrm{H}, \mathrm{FcH}), 4.06\left(\mathrm{t}, J=6.5 \mathrm{~Hz}, 2 \mathrm{H}, \mathrm{OCH}_{2}\right), 1.84-1.80(\mathrm{~m}$, 2H, $\left.\mathrm{CH}_{2}\right), 1.49-1.46\left(\mathrm{~m}, 2 \mathrm{H}, \mathrm{CH}_{2}\right), 1.37-1.20\left(\mathrm{~m}, 16 \mathrm{H}, \mathrm{CH}_{2}\right), 0.88$ $\left(\mathrm{t}, J=6.5 \mathrm{~Hz}, 3 \mathrm{H}, \mathrm{CH}_{3}\right) ;{ }^{13} \mathrm{C} \mathrm{NMR}\left(125 \mathrm{MHz}, \mathrm{CDCl}_{3}\right)(\delta \mathrm{ppm})$ : 188.98, 164.59, 163.78, 154.57, 153.72, 150.19, 146.41, 132.41, $129.55,124.45,122.99,122.61,121.13,114.41,71.17,69.97$, 69.63, 68.40, 31.94, 29.63, 29.37, 22.71, 14.14; HRMS, MALDI ${ }^{+}$, $m / z$ : calcd for $\mathrm{C}_{44} \mathrm{H}_{48} \mathrm{FeN}_{2} \mathrm{O}_{4}:\left[\mathrm{M}^{+}\right]$724.2964, found: 724.2966 .

Ia-14. Yield $72.3 \%, \mathrm{mp} 172-176{ }^{\circ} \mathrm{C}, R_{\mathrm{f}}=0.27\left(\mathrm{CH}_{2} \mathrm{Cl}_{2}\right.$ : petroleum ether $=3: 1)$; IR $\left(\mathrm{KBr}, \nu, \mathrm{cm}^{-1}\right): 3094,2921,2849,1732$, 1654, 1590, 1512, 1316, 1269, 1077, 977; ${ }^{1} \mathrm{H}$ NMR $(500 \mathrm{MHz}$, $\left.\mathrm{CDCl}_{3}\right)(\delta \mathrm{ppm}): 8.16\left(\mathrm{~d}, J=8.5 \mathrm{~Hz}, 2 \mathrm{H}, \mathrm{C}_{6} \mathrm{H}_{4}\right), 8.13(\mathrm{~d}, J=8.0 \mathrm{~Hz}$, $\left.2 \mathrm{H}, \mathrm{C}_{6} \mathrm{H}_{4}\right), 8.05\left(\mathrm{~d}, J=8.5 \mathrm{~Hz}, 2 \mathrm{H}, \mathrm{C}_{6} \mathrm{H}_{4}\right), 8.01(\mathrm{~d}, J=8.0 \mathrm{~Hz}, 2 \mathrm{H}$, $\left.\mathrm{C}_{6} \mathrm{H}_{4}\right), 7.79$ (d, $\left.J=15.0 \mathrm{~Hz}, 1 \mathrm{H}, \mathrm{CH}\right), 7.40(\mathrm{~d}, J=8.5 \mathrm{~Hz}, 2 \mathrm{H}$, $\left.\mathrm{C}_{6} \mathrm{H}_{4}\right), 7.17$ (d, $\left.J=15.0 \mathrm{~Hz}, 1 \mathrm{H}, \mathrm{CH}\right), 6.98$ (d, $J=8.5 \mathrm{~Hz}, 2 \mathrm{H}$, $\left.\mathrm{C}_{6} \mathrm{H}_{4}\right), 4.66(\mathrm{~s}, 2 \mathrm{H}, \mathrm{FcH}), 4.55(\mathrm{~s}, 2 \mathrm{H}, \mathrm{FcH}), 4.23(\mathrm{~s}, 5 \mathrm{H}, \mathrm{FcH}), 4.06$ $\left(\mathrm{t}, J=6.5 \mathrm{~Hz}, 2 \mathrm{H}, \mathrm{OCH}_{2}\right), 1.86-1.80\left(\mathrm{~m}, 2 \mathrm{H}, \mathrm{CH}_{2}\right), 1.49-1.45(\mathrm{~m}$, $\left.2 \mathrm{H}, \mathrm{CH}_{2}\right), 1.37-1.26\left(\mathrm{~m}, 20 \mathrm{H}, \mathrm{CH}_{2}\right), 0.88\left(\mathrm{t}, J=6.5 \mathrm{~Hz}, 3 \mathrm{H}, \mathrm{CH}_{3}\right)$; ${ }^{13} \mathrm{C}$ NMR (125 $\mathrm{MHz}, \mathrm{CDCl}_{3}$ ) ( $\left.\delta \mathrm{ppm}\right):$ 189.00, 164.59, 163.78, 154.58, 153.71, 150.18, 147.55, 132.41, 129.45, 124.45, 122.93, 122.61, 119.12, 114.41, 70.51, 69.63, 69.34, 68.40, 31.94, 29.64, 29.38, 29.11, 26.00, 22.71, 14.15; HRMS, MALDI ${ }^{+}, \mathrm{m} / \mathrm{z}$ : calcd for $\mathrm{C}_{46} \mathrm{H}_{52} \mathrm{FeN}_{2} \mathrm{O}_{4}:\left[\mathrm{M}^{+}\right]$752.3277, found: 752.3278 .

Ia-16. Yield $73 \%, \mathrm{mp} 168-170{ }^{\circ} \mathrm{C}, R_{\mathrm{f}}=0.275\left(\mathrm{CH}_{2} \mathrm{Cl}_{2}\right.$ : petroleum ether $=3: 1)$; IR (KBr, $\left.\nu, \mathrm{cm}^{-1}\right)$ : 3094, 2950, 2920, 2854, 1732, 1653, 1592, 1509, 1316, 1260, 1074, 979; ${ }^{1} \mathrm{H}$ NMR (500 $\left.\mathrm{MHz} \mathrm{CDCl}_{3}\right)(\delta \mathrm{ppm}): 8.16\left(\mathrm{~d}, J=8.5 \mathrm{~Hz}, 2 \mathrm{H}, \mathrm{C}_{6} \mathrm{H}_{4}\right), 8.11(\mathrm{~b}, 2 \mathrm{H}$, $\mathrm{C}_{6} \mathrm{H}_{4}$ ), 7.05 (d, $\left.J=8.5 \mathrm{~Hz}, 2 \mathrm{H}, \mathrm{C}_{6} \mathrm{H}_{4}\right), 8.01$ (b, 2H, $\mathrm{C}_{6} \mathrm{H}_{4}$ ), 7.71 (b, $1 \mathrm{H}, \mathrm{CH}), 7.40$ (d, J = 8.5 Hz, 2H, $\left.\mathrm{C}_{6} \mathrm{H}_{4}\right), 7.14(\mathrm{~s}, 1 \mathrm{H}, \mathrm{CH}), 6.98$ (d,
$\left.J=8.5 \mathrm{~Hz}, 2 \mathrm{H}, \mathrm{C}_{6} \mathrm{H}_{4}\right), 4.84(\mathrm{~s}, 2 \mathrm{H}, \mathrm{FcH}), 4.71(\mathrm{~s}, 2 \mathrm{H}, \mathrm{FcH}), 4.39(\mathrm{~s}$, $5 \mathrm{H}, \mathrm{FcH}), 4.05$ (t $\left.J=6.5 \mathrm{~Hz}, 2 \mathrm{H}, \mathrm{OCH}_{2}\right), 1.85-1.80\left(\mathrm{~m}, 2 \mathrm{H}, \mathrm{CH}_{2}\right)$, 1.49-1.46 (m, 2H, $\left.\mathrm{CH}_{2}\right), 1.37-1.20\left(\mathrm{~m}, 24 \mathrm{H}, \mathrm{CH}_{2}\right), 0.88(\mathrm{t}, J=$ $\left.6.7 \mathrm{~Hz}, 3 \mathrm{H}, \mathrm{CH}_{3}\right) ;{ }^{13} \mathrm{C} \mathrm{NMR}\left(125 \mathrm{MHz}, \mathrm{CDCl}_{3}\right)(\delta \mathrm{ppm}): 188.94$, $164.58,163.78,154.57,153.72$, 151.74-151.56, 150.18-149.98, $132.41,124.45,122.98,122.61,121.13,114.41,68.41,31.95$, 29.71, 29.64, 29.38, 26.00, 22.71, 14.15; HRMS, MALDI ${ }^{+}, m / z$ : calcd for $\mathrm{C}_{48} \mathrm{H}_{56} \mathrm{FeN}_{2} \mathrm{O}_{4}$ : $\left[\mathrm{M}^{+}\right]$780.3590, found: 780.3585 .

Ib-8. Yield $77.2 \%, \mathrm{mp} 142-145{ }^{\circ} \mathrm{C}, R_{\mathrm{f}}=0.23\left(\mathrm{CH}_{2} \mathrm{Cl}_{2}\right.$ : petroleum ether $=3: 1)$; IR ( $\left.\mathrm{KBr}, \nu, \mathrm{cm}^{-1}\right): 3078.37,2926.80,2852.78$, $1731.00,1650.52$, 1603.11, 1509.38, 1260.60, 1070.15, 976.68; ${ }^{1} \mathrm{H}$ NMR (500 MHz, $\mathrm{CDCl}_{3}$ ) ( $\left.\delta \mathrm{ppm}\right): 8.17\left(\mathrm{~d}, J=8.5 \mathrm{~Hz}, 2 \mathrm{H}, \mathrm{C}_{6} \mathrm{H}_{4}\right)$, $8.11\left(\mathrm{~d}, J=8.5 \mathrm{~Hz}, 2 \mathrm{H}, \mathrm{C}_{6} \mathrm{H}_{4}\right), 8.05\left(\mathrm{~d}, J=8.5 \mathrm{~Hz}, 2 \mathrm{H}, \mathrm{C}_{6} \mathrm{H}_{4}\right), 8.00$ (s, $\left.2 \mathrm{H}, \mathrm{C}_{6} \mathrm{H}_{4}\right), 7.82(\mathrm{~b}, 1 \mathrm{H}, \mathrm{CH}), 7.40\left(\mathrm{~d}, J=8.5 \mathrm{~Hz}, 2 \mathrm{H}, \mathrm{C}_{6} \mathrm{H}_{4}\right)$, 7.05 (b, 1H, CH), 6.99 (d, J=8.5 Hz, 2H, $\left.\mathrm{C}_{6} \mathrm{H}_{4}\right), 4.65$ (b, 2H, FcH), 4.51-4.29 (m, 3H, FcH), 4.17-4.04 (m, 3H, FcH, $\left.\mathrm{OCH}_{2}\right), 3.93$ (s, $1 \mathrm{H}, \mathrm{FcH}), 2.02-1.62\left(\mathrm{~m}, 8 \mathrm{H}, \mathrm{CH}_{2}\right), 1.52-1.45\left(\mathrm{~m}, 2 \mathrm{H}, \mathrm{CH}_{2}\right), 1.42-$ $1.24\left(\mathrm{~m}, 8 \mathrm{H}, \mathrm{CH}_{2}\right), 0.90\left(\mathrm{t}, J=6.5 \mathrm{~Hz}, 3 \mathrm{H}, \mathrm{CH}_{3}\right) ;{ }^{13} \mathrm{C} \mathrm{NMR}(125$ $\left.\mathrm{MHz}, \mathrm{CDCl}_{3}\right)(\delta \mathrm{ppm}): 188.66,164.58,163.77,162.62,154.38$, $153.66,150.20,149.61,147.76,147.73,147.70,132.41,132.41$, 131.16, 129.31, 125.80, 124.48, 124.41, 123.11, 123.03, 122.60, 121.13, 114.41, 89.67, 86.60, 73.49, 73.47, 70.78, 69.99, 68.40, $34.85,31.82,29.71,29.34,29.24,29.01,26.00,24.54,24.09$, 22.67, 14.13; HRMS, ESI $\mathrm{m} / z$ : calcd for $\mathrm{C}_{43} \mathrm{H}_{44} \mathrm{FeN}_{2} \mathrm{O}_{4}:[\mathrm{M}]^{+}$ 708.2646, found: 708.2631 .

Ib-12. Yield 78.5\%, mp 140-144 ${ }^{\circ} \mathrm{C}, \quad R_{\mathrm{f}}=0.215$ $\left(\mathrm{CH}_{2} \mathrm{Cl}_{2}\right.$ : petroleum ether $\left.=3: 1\right)$; IR $\left(\mathrm{KBr}, \nu, \mathrm{cm}^{-1}\right): 3124.18$, 2923.63, 2853.37, 1727.16, 1648.34, 1602.12, 1510.01, 1258.84, 1075.98, 975.69. ${ }^{1} \mathrm{H}$ NMR (500 $\left.\mathrm{MHz}, \mathrm{CDCl}_{3}\right)(\delta \mathrm{ppm}): 8.16(\mathrm{~d}, J=$ $\left.8.5 \mathrm{~Hz}, 2 \mathrm{H}, \mathrm{C}_{6} \mathrm{H}_{4}\right), 8.11\left(\mathrm{~d}, J=6.5 \mathrm{~Hz}, 2 \mathrm{H}, \mathrm{C}_{6} \mathrm{H}_{4}\right), 8.06(\mathrm{~d}, J=$ $\left.8.5 \mathrm{~Hz}, 2 \mathrm{H}, \mathrm{C}_{6} \mathrm{H}_{4}\right), 8.00\left(\mathrm{~d}, J=7.0 \mathrm{~Hz}, 2 \mathrm{H}, \mathrm{C}_{6} \mathrm{H}_{4}\right), 7.76(\mathrm{~d}, J=$ $14.0 \mathrm{~Hz}, 1 \mathrm{H}, \mathrm{CH}), 7.41\left(\mathrm{~d}, J=8.5 \mathrm{~Hz}, 2 \mathrm{H}, \mathrm{C}_{6} \mathrm{H}_{4}\right), 7.05(\mathrm{~d}, J=$ $14.0 \mathrm{~Hz}, 1 \mathrm{H}, \mathrm{CH}), 7.00\left(\mathrm{~d}, J=9.0 \mathrm{~Hz}, 2 \mathrm{H}, \mathrm{C}_{6} \mathrm{H}_{4}\right), 4.54(\mathrm{~s}, 2 \mathrm{H}$, $\mathrm{FcH}), 4.36(\mathrm{~s}, 1 \mathrm{H}, \mathrm{FcH}), 4.33(\mathrm{~s}, 1 \mathrm{H}, \mathrm{FcH}), 4.28(\mathrm{~s}, 1 \mathrm{H}, \mathrm{FcH}), 4.07$ $\left(\mathrm{t}, J=6.5 \mathrm{~Hz}, 3 \mathrm{H}, \mathrm{OCH}_{2}\right), 3.90(\mathrm{~s}, 1 \mathrm{H}, \mathrm{FcH}), 2.06-1.89(\mathrm{~m}, 6 \mathrm{H}$, $\mathrm{CH}_{2}$ ), 1.86-1.80 (m, 2H, $\mathrm{CH}_{2}$ ), 1.51-1.45 (m, 2H, $\left.\mathrm{CH}_{2}\right), 1.37-1.25$ $\left(\mathrm{m}, 16 \mathrm{H}, \mathrm{CH}_{2}\right), 0.89\left(\mathrm{t}, J=6.5 \mathrm{~Hz}, 3 \mathrm{H}, \mathrm{CH}_{3}\right) ;{ }^{13} \mathrm{C} \mathrm{NMR}(125 \mathrm{MHz}$, $\left.\mathrm{CDCl}_{3}\right)(\delta \mathrm{ppm}): 189.04,164.53,163.79,154.46,153.70,150.24$, $147.64,147.64,140.56,132.39,129.30,124.39,122.89,122.55$, 114.43, 89.63, 86.58, 73.44, 73.43, 70.80, 70.24, 70.22, 70.20, $70.06,68.41,34.83,31.91,29.61,29.35,29.10,25.99,24.55$, 24.11, 22.67, 14.08; HRMS, ESI $m / z$ : calcd for $\mathrm{C}_{47} \mathrm{H}_{52} \mathrm{FeN}_{2} \mathrm{O}_{4}$ : $[\mathrm{M}]^{+}$764.3272, found: 764.3263 .

Ic-8. Yield $72.5 \%, \mathrm{mp} 158-165^{\circ} \mathrm{C}, R_{\mathrm{f}}=0.25\left(\mathrm{CH}_{2} \mathrm{Cl}_{2}\right.$ : petroleum ether $=2: 1)$; IR (KBr, $\left.\nu, \mathrm{cm}^{-1}\right): 3052,2953,2922,2853,1729$, 1656, 1605, 1573, 1466, 1306, 1259, 1066, 842; ${ }^{1} \mathrm{H}$ NMR (500 $\left.\mathrm{MHz}, \mathrm{CDCl}_{3}\right)(\delta \mathrm{ppm}): 8.19\left(\mathrm{~d}, J=8.5 \mathrm{~Hz}, 2 \mathrm{H}, \mathrm{C}_{6} \mathrm{H}_{4}\right), 8.17(\mathrm{~d}, J=$ $\left.9.0 \mathrm{~Hz}, 2 \mathrm{H}, \mathrm{C}_{6} \mathrm{H}_{4}\right), 8.07$ (d, $\left.J=8.5 \mathrm{~Hz}, 2 \mathrm{H}, \mathrm{C}_{6} \mathrm{H}_{4}\right), 8.03(\mathrm{~d}, J=$ $\left.8.5 \mathrm{~Hz}, 2 \mathrm{H}, \mathrm{C}_{6} \mathrm{H}_{4}\right), 7.86(\mathrm{~d}, J=15.5 \mathrm{~Hz}, 1 \mathrm{H}, \mathrm{CH}), 7.69-7.68(\mathrm{~m}$, $\left.2 \mathrm{H}, \mathrm{C}_{6} \mathrm{H}_{5}\right), 7.58(\mathrm{~d}, J=15.5 \mathrm{~Hz}, 1 \mathrm{H}, \mathrm{CH}), 7.45-7.44(\mathrm{~m}, 3 \mathrm{H}$, $\left.\mathrm{C}_{6} \mathrm{H}_{5}\right), 7.42\left(\mathrm{~d}, J=9.0 \mathrm{~Hz}, 2 \mathrm{H}, \mathrm{C}_{6} \mathrm{H}_{4}\right), 6.99(\mathrm{~d}, J=8.5 \mathrm{~Hz}, 2 \mathrm{H}$, $\left.\mathrm{C}_{6} \mathrm{H}_{4}\right), 4.06\left(\mathrm{t}, J=6.5 \mathrm{~Hz}, 2 \mathrm{H}, \mathrm{OCH}_{2}\right), 1.86-1.81\left(\mathrm{~m}, 2 \mathrm{H}, \mathrm{CH}_{2}\right)$, 1.51-1.47 (m, 2H, $\left.\mathrm{CH}_{2}\right), 1.42-1.23\left(\mathrm{~m}, 8 \mathrm{H}, \mathrm{CH}_{2}\right), 0.90(\mathrm{t}, J=$ $\left.7.0 \mathrm{~Hz}, 3 \mathrm{H}, \mathrm{CH}_{3}\right) ;{ }^{13} \mathrm{C} \mathrm{NMR}\left(125 \mathrm{MHz}, \mathrm{CDCl}_{3}\right)(\delta \mathrm{ppm}): 189.85$, $164.58,163.79,154.83,153.79,150.17,145.34,139.69,134.81$, $132.41,130.75,129.59,129.04,128.57,124.49,123.01,122.62$, $121.99,121.12$, 114.42, 68.40, 31.82, 29.34, 29.23, 29.11, 26.00, 
22.67, 14.12; HRMS, MALDI ${ }^{+} m / z$ : calcd for $\mathrm{C}_{36} \mathrm{H}_{37} \mathrm{~N}_{2} \mathrm{NaO}_{4}$ : [M+ $\mathrm{Na}]^{+}$583.2567, found: 583.2560 .

Ic-12. Yield $75.3 \%, \mathrm{mp} 150-156{ }^{\circ} \mathrm{C}, R_{\mathrm{f}}=0.277\left(\mathrm{CH}_{2} \mathrm{Cl}_{2}\right.$ : petroleum ether $=2: 1)$; IR $\left(\mathrm{KBr}, \nu, \mathrm{cm}^{-1}\right): 3052,2953,2821,2853$, 1729, 1656, 1605, 1578, 1495, 1306, 1259, 1066, 842; ${ }^{1} \mathrm{H}$ NMR $\left(500 \mathrm{MHz}, \mathrm{CDCl}_{3}\right)(\delta \mathrm{ppm}): 8.18\left(\mathrm{~d}, J=8.5 \mathrm{~Hz}, 2 \mathrm{H}, \mathrm{C}_{6} \mathrm{H}_{4}\right), 8.16(\mathrm{~d}$, $\left.J=9.0 \mathrm{~Hz}, 2 \mathrm{H}, \mathrm{C}_{6} \mathrm{H}_{4}\right), 8.05\left(\mathrm{~d}, J=9.0 \mathrm{~Hz}, 2 \mathrm{H}, \mathrm{C}_{6} \mathrm{H}_{4}\right), 8.02(\mathrm{~d}, J=$ $\left.8.5 \mathrm{~Hz}, 2 \mathrm{H}, \mathrm{C}_{6} \mathrm{H}_{4}\right), 7.85(\mathrm{~d}, J=15.5 \mathrm{~Hz}, 1 \mathrm{H}, \mathrm{CH}), 7.69-7.68(\mathrm{~m}$, $\left.2 \mathrm{H}, \mathrm{C}_{6} \mathrm{H}_{5}\right), 7.58(\mathrm{~d}, J=15.5 \mathrm{~Hz}, 1 \mathrm{H}, \mathrm{CH}), 7.45-7.44(\mathrm{~m}, 3 \mathrm{H}$, $\left.\mathrm{C}_{6} \mathrm{H}_{5}\right), 7.42\left(\mathrm{~d}, J=9.0 \mathrm{~Hz}, 2 \mathrm{H}, \mathrm{C}_{6} \mathrm{H}_{4}\right), 7.00(\mathrm{~d}, J=9.0 \mathrm{~Hz}, 2 \mathrm{H}$, $\left.\mathrm{C}_{6} \mathrm{H}_{4}\right), 4.06\left(\mathrm{t}, J=6.5 \mathrm{~Hz}, 2 \mathrm{H}, \mathrm{OCH}_{2}\right), 1.86-1.80\left(\mathrm{~m}, 2 \mathrm{H}, \mathrm{CH}_{2}\right)$, 1.48-1.45 (m, $\left.2 \mathrm{H}, \mathrm{CH}_{2}\right), 1.37-1.26\left(\mathrm{~m}, 16 \mathrm{H}, \mathrm{CH}_{2}\right), 0.89(\mathrm{t}, J=$ $\left.7.0 \mathrm{~Hz}, 3 \mathrm{H}, \mathrm{CH}_{3}\right) ;{ }^{13} \mathrm{C}$ NMR $\left(125 \mathrm{MHz}, \mathrm{CDCl}_{3}\right)(\delta \mathrm{ppm}): 189.84$, $164.57,163.79,154.82,153.79,150.16,145.34,139.68,134.81$, $132.41,130.75,129.59,129.04,128.58,124.49,123.01,122.62$, $121.98,121.12,114.42,68.40,31.94,29.67,29.65,29.60,29.57$, 29.37, 29.11, 26.00, 22.71, 14.15; HRMS, MALDI $^{+} \mathrm{m} / \mathrm{z}$ : calcd for $\mathrm{C}_{40} \mathrm{H}_{45} \mathrm{~N}_{2} \mathrm{O}_{4}:[\mathrm{M}+\mathrm{H}]^{+}$617.3379, found: 617.3350. Elemental analysis: calcd for $\mathrm{C}_{40} \mathrm{H}_{44} \mathrm{~N}_{2} \mathrm{O}_{4}$ : C 77.89, $\mathrm{H} 7.19, \mathrm{~N} 4.54 \%$; found: C 77.73, H 7.55, N 4.33\%.

Ic-14. Yield $76.9 \%, \mathrm{mp} 162-164{ }^{\circ} \mathrm{C}, R_{\mathrm{f}}=0.28$ (ethyl acetate : petroleum ether $=1: 20)$; IR $\left(\mathrm{KBr}, \nu, \mathrm{cm}^{-1}\right)$ : 3125.77, 2954.43, 2849.44, 1736.54, 1657.86, 1608.91, 1575.47, 1497.17, 1307.46, $1259.58,1082.75,842.04 ;{ }^{1} \mathrm{H}$ NMR $\left(500 \mathrm{MHz}, \mathrm{CDCl}_{3}\right)(\delta$ ppm): 8.18 (d, $\left.J=9.0 \mathrm{~Hz}, 2 \mathrm{H}, \mathrm{C}_{6} \mathrm{H}_{4}\right), 8.16(\mathrm{~d}, J=9.0 \mathrm{~Hz}, 2 \mathrm{H}$, $\left.\mathrm{C}_{6} \mathrm{H}_{4}\right), 8.06\left(\mathrm{t}, J=9.0 \mathrm{~Hz}, 2 \mathrm{H}, \mathrm{C}_{6} \mathrm{H}_{4}\right), 8.04(\mathrm{t}, J=8.5 \mathrm{~Hz}, 2 \mathrm{H}$, $\left.\mathrm{C}_{6} \mathrm{H}_{4}\right), 7.88(\mathrm{~d}, J=15.5 \mathrm{~Hz}, 1 \mathrm{H}, \mathrm{CH}), 7.69-7.68\left(\mathrm{~m}, 2 \mathrm{H}, \mathrm{C}_{6} \mathrm{H}_{5}\right)$, $7.60(\mathrm{~d}, J=15.5 \mathrm{~Hz}, 1 \mathrm{H}, \mathrm{ArH}), 7.42-7.44\left(\mathrm{~m}, 3 \mathrm{H}, \mathrm{C}_{6} \mathrm{H}_{5}\right), 7.40(\mathrm{~d}, J$ $\left.=9.0 \mathrm{~Hz}, \mathrm{C}_{6} \mathrm{H}_{4}\right), 7.00\left(\mathrm{~d}, J=9.0 \mathrm{~Hz}, 2 \mathrm{H}, \mathrm{C}_{6} \mathrm{H}_{4}\right), 4.06(\mathrm{t}, J=6.5 \mathrm{~Hz}$, $\left.2 \mathrm{H}, \mathrm{OCH}_{2}\right), 1.86-1.80\left(\mathrm{~m}, 2 \mathrm{H}, \mathrm{CH}_{2}\right), 1.51-1.45\left(\mathrm{~m}, 2 \mathrm{H}, \mathrm{CH}_{2}\right)$, 1.37-1.26 (m, 20H, $\left.\mathrm{CH}_{2}\right), 0.88\left(\mathrm{t}, J=7.0 \mathrm{~Hz}, 3 \mathrm{H}, \mathrm{CH}_{3}\right) ;{ }^{13} \mathrm{C} \mathrm{NMR}$ $\left(125 \mathrm{MHz}, \mathrm{CDCl}_{3}\right)(\delta \mathrm{ppm}): 189.86,164.58,163.79,153.79$, $145.35,139.69,134.80,132.42,130.75,129.59,129.04,128.58$, $124.49,123.01,122.63,121.98,114.42,68.40,31.94,29.71$, 29.69, 29.61, 29.38, 29.11, 26.00, 22.71, 14.15; HRMS, MALDI ${ }^{+}$ $m / z$ : calcd for $\mathrm{C}_{42} \mathrm{H}_{49} \mathrm{~N}_{2} \mathrm{O}_{4}:[\mathrm{M}+\mathrm{H}]^{+}$645.3692, found: 645.3668.

Id-8. Yield $75.7 \%$, mp $176-179{ }^{\circ} \mathrm{C}, R_{\mathrm{f}}=0.27\left(\mathrm{CH}_{2} \mathrm{Cl}_{2}\right.$ : petroleum ether = 2:1); IR $\left(\mathrm{KBr}, \nu, \mathrm{cm}^{-1}\right): 3105,2925,2853,1731$, $1647,1602,1567,1494,1315,1257,1074,844 ;{ }^{1} \mathrm{H}$ NMR (500 $\left.\mathrm{MHz}, \mathrm{CDCl}_{3}\right)(\delta \mathrm{ppm}): 8.17\left(\mathrm{~d}, J=8.5 \mathrm{~Hz}, 4 \mathrm{H}, \mathrm{C}_{6} \mathrm{H}_{4}\right), 8.05(\mathrm{~d}, J=$ $\left.8.5 \mathrm{~Hz}, 2 \mathrm{H}, \mathrm{C}_{6} \mathrm{H}_{4}\right), 8.01\left(\mathrm{~d}, J=8.5 \mathrm{~Hz}, 2 \mathrm{H}, \mathrm{C}_{6} \mathrm{H}_{4}\right), 7.98(\mathrm{~d}, J=$ $15.5 \mathrm{~Hz}, 1 \mathrm{H}, \mathrm{CH}), 7.46(\mathrm{~d}, J=5.0 \mathrm{~Hz}, 1 \mathrm{H}, \mathrm{TpH}), 7.41-7.37(\mathrm{~m}$, $\left.4 \mathrm{H}, \mathrm{C}_{6} \mathrm{H}_{4}\right), 7.12(\mathrm{~m}, 1 \mathrm{H}, \mathrm{TpH}), 6.99\left(\mathrm{~d}, J=8.5 \mathrm{~Hz}, 2 \mathrm{H}, \mathrm{C}_{6} \mathrm{H}_{4}\right), 4.06$ $\left(\mathrm{t}, J=6.5 \mathrm{~Hz}, 2 \mathrm{H}, \mathrm{CH}_{2}\right), 1.87-1.80\left(\mathrm{~m}, 2 \mathrm{H}, \mathrm{CH}_{2}\right), 1.50-1.46(\mathrm{~m}$, $\left.2 \mathrm{H}, \mathrm{CH}_{2}\right), 1.41-1.24\left(\mathrm{~m}, 8 \mathrm{H}, \mathrm{CH}_{2}\right), 0.90\left(\mathrm{t}, J=7.0 \mathrm{~Hz}, 3 \mathrm{H}, \mathrm{CH}_{3}\right)$; ${ }^{13} \mathrm{C}$ NMR $\left(125 \mathrm{MHz}, \mathrm{CDCl}_{3}\right)(\delta \mathrm{ppm}): 189.17,164.56,163.79$, 154.81, 153.78, 152.56, 140.34, 137.68, 132.41, 129.48, 129.12, $128.45,124.49,123.01,122.62,114.42,68.40,31.82,29.34$, 29.24, 29.11, 26.00, 22.67, 14.12; HRMS, $\mathrm{MALDI}^{+} \mathrm{m} / z$ : calcd for $\mathrm{C}_{34} \mathrm{H}_{35} \mathrm{~N}_{2} \mathrm{O}_{4} \mathrm{~S}:[\mathrm{M}+\mathrm{H}]^{+}$567.2318, found: 567.2299.

Id-12. Yield $76 \%, \mathrm{mp} 149-158{ }^{\circ} \mathrm{C}, R_{\mathrm{f}}=0.29\left(\mathrm{CH}_{2} \mathrm{Cl}_{2}\right.$ : petroleum ether =2:1); IR $\left(\mathrm{KBr}, \nu, \mathrm{cm}^{-1}\right): 3128,2954,2919,2849$, 1736, 1652, 1596, 1510, 1469, 1289, 1260, 1081, 844; ${ }^{1} \mathrm{H}$ NMR $\left(500 \mathrm{MHz}, \mathrm{CDCl}_{3}\right)(\delta \mathrm{ppm}): 8.17\left(\mathrm{~d}, J=8.5 \mathrm{~Hz}, 4 \mathrm{H}, \mathrm{C}_{6} \mathrm{H}_{4}\right), 8.05(\mathrm{~d}$, $\left.J=8.5 \mathrm{~Hz}, 2 \mathrm{H}, \mathrm{C}_{6} \mathrm{H}_{4}\right), 8.02\left(\mathrm{~d}, J=8.5 \mathrm{~Hz}, 2 \mathrm{H}, \mathrm{C}_{6} \mathrm{H}_{4}\right), 7.98(\mathrm{~d}, J=$ $15.5 \mathrm{~Hz}, 1 \mathrm{H}, \mathrm{CH}), 7.45(\mathrm{~d}, J=5 \mathrm{~Hz}, 1 \mathrm{H}, \mathrm{TpH}), 7.41-7.37(\mathrm{~m}, 4 \mathrm{H}$, $\left.\mathrm{CH}, \mathrm{TpH}, \mathrm{C}_{6} \mathrm{H}_{4}\right), 7.12(\mathrm{t}, J=4.5 \mathrm{~Hz}, 1 \mathrm{H}, \mathrm{TpH}), 7.00(\mathrm{~d}, J=9.0 \mathrm{~Hz}$, $\left.2 \mathrm{H}, \mathrm{C}_{6} \mathrm{H}_{4}\right), 4.05\left(\mathrm{t}, J=6.5 \mathrm{~Hz}, 2 \mathrm{H}, \mathrm{OCH}_{2}\right), 1.85-1.80(\mathrm{~m}, 2 \mathrm{H}$, $\left.\mathrm{CH}_{2}\right), 1.51-1.45\left(\mathrm{~m}, 2 \mathrm{H}, \mathrm{CH}_{2}\right), 1.37-1.27\left(\mathrm{~m}, 16 \mathrm{H}, \mathrm{CH}_{2}\right), 0.89(\mathrm{t}$, $\left.J=7.0 \mathrm{~Hz}, 3 \mathrm{H}, \mathrm{CH}_{3}\right) ;{ }^{13} \mathrm{C}$ NMR $\left(125 \mathrm{MHz}, \mathrm{CDCl}_{3}\right)(\delta \mathrm{ppm})$ : 189.15, 164.57, 163.79, 154.81, 153.78, 150.16, 140.34, 139.61, 137.66, 132.41, 129.48, 129.12, 128.46, 124.48, 123.01, 122.61, 121.12, 120.65, 114.41, 68.40, 31.94, 29.67, 29.61, 29.58, 29.37, 29.11, 26.00, 22.71, 14.14; HRMS, MALDI $^{+} \mathrm{m} / \mathrm{z}$ : calcd for $\mathrm{C}_{38} \mathrm{H}_{43} \mathrm{~N}_{2} \mathrm{O}_{4} \mathrm{~S}:[\mathrm{M}+\mathrm{H}]^{+}$623.2944, found 623.2926; elemental analysis: calcd for $\mathrm{C}_{38} \mathrm{H}_{42} \mathrm{~N}_{2} \mathrm{O}_{4} \mathrm{~S}$ : C 73.28, $\mathrm{H} 6.80, \mathrm{~N} 4.50 \%$; found: C $72.89, \mathrm{H} 6.76, \mathrm{~N} 4.43 \%$.

Id-14. Yield $73 \%$, mp $158-160{ }^{\circ} \mathrm{C}, R_{\mathrm{f}}=0.305\left(\mathrm{CH}_{2} \mathrm{Cl}_{2}\right.$ : petroleum ether = 2:1); IR $\left(\mathrm{KBr}, \nu, \mathrm{cm}^{-1}\right): 3078,2954,2918,2849$, $1735,1652,1596,1510,1469,1290,1259,1081,852 ;{ }^{1} \mathrm{H}$ NMR $\left(500 \mathrm{MHz}, \mathrm{CDCl}_{3}\right)(\delta \mathrm{ppm}): 8.17\left(\mathrm{~d}, J=8.5 \mathrm{~Hz}, 4 \mathrm{H}, \mathrm{C}_{6} \mathrm{H}_{4}\right), 8.05$ $\left(\mathrm{d}, J=8.5 \mathrm{~Hz}, 2 \mathrm{H}, \mathrm{C}_{6} \mathrm{H}_{4}\right), 8.02\left(\mathrm{~d}, J=8.5 \mathrm{~Hz}, 2 \mathrm{H}, \mathrm{C}_{6} \mathrm{H}_{4}\right), 7.98(\mathrm{~d}$, $J=15.5 \mathrm{~Hz}, 1 \mathrm{H}, \mathrm{CH}), 7.45(\mathrm{~d}, J=5 \mathrm{~Hz}, 1 \mathrm{H}, \mathrm{TpH}), 7.41-7.37(\mathrm{~m}$, $\left.4 \mathrm{H}, \mathrm{CH}, \mathrm{TpH}, \mathrm{C}_{6} \mathrm{H}_{4}\right), 7.12(\mathrm{t}, J=4.5 \mathrm{~Hz}, 1 \mathrm{H}, \mathrm{TpH}), 7.00(\mathrm{~d}, J=$ $\left.9.0 \mathrm{~Hz}, 2 \mathrm{H}, \mathrm{C}_{6} \mathrm{H}_{4}\right), 4.06(\mathrm{t}, J=6.5 \mathrm{~Hz}, 2 \mathrm{H}, \mathrm{ArH}), 1.86-1.80(\mathrm{~m}, 2 \mathrm{H}$, $\left.\mathrm{CH}_{2}\right), 1.49-1.47\left(\mathrm{~m}, 2 \mathrm{H}, \mathrm{CH}_{2}\right), 1.37-1.27\left(\mathrm{~m}, 20 \mathrm{H}, \mathrm{CH}_{2}\right), 0.88(\mathrm{t}$, $\left.J=7.0 \mathrm{~Hz}, 3 \mathrm{H}, \mathrm{CH}_{3}\right) ;{ }^{13} \mathrm{C}$ NMR $\left(125 \mathrm{MHz}, \mathrm{CDCl}_{3}\right)(\delta \mathrm{ppm})$ : 189.16, 164.58, 163.78, 154.80, 153.78, 150.16, 140.34, 137.68, 132.41, 129.49, 129.13, 128.46, 124.49, 123.01, 122.62, 120.64, 114.41, 68.40, 31.95, 29.69, 29.67, 29.61, 29.57, 29.38, 29.11, 26.00, 22.72, 14.15; HRMS, MALDI ${ }^{+} \mathrm{m} / \mathrm{z}$ : calcd for $\mathrm{C}_{40} \mathrm{H}_{47} \mathrm{~N}_{2} \mathrm{O}_{4} \mathrm{~S}$ : $[\mathrm{M}+\mathrm{H}]^{+}$651.3257, found: 651.3243; elemental analysis: calcd for $\mathrm{C}_{40} \mathrm{H}_{46} \mathrm{~N}_{2} \mathrm{O}_{4} \mathrm{~S}: \mathrm{C} 73.81 \%, \mathrm{H} 7.12, \mathrm{~N} 4.30 \%$; found: $\mathrm{C} 73.65, \mathrm{H}$ 7.11, $\mathrm{N} 4.24 \%$.

IIc-8. Yield $53.1 \%, \mathrm{mp} 71-73{ }^{\circ} \mathrm{C}, R_{\mathrm{f}}=0.2\left(\mathrm{CH}_{2} \mathrm{Cl}_{2}\right.$ : petroleum ether : ethyl acetate $=2 \mathrm{~mL}: 1 \mathrm{~mL}: 20 \mu \mathrm{L})$; IR $\left(\mathrm{KBr}, \nu, \mathrm{cm}^{-1}\right)$ : 3066, 2923, 2853, 2360, 1729, 1656, 1595, 1569, 1496, 1193, 1033, 983; ${ }^{1} \mathrm{H}$ NMR $\left(500 \mathrm{MHz}, \mathrm{CDCl}_{3}\right)(\delta \mathrm{ppm}): 8.19(\mathrm{~d}, J=$ $8.5 \mathrm{~Hz}, 2 \mathrm{H}), 8.06(\mathrm{~d}, J=8.5 \mathrm{~Hz}, 2 \mathrm{H}), 8.04(\mathrm{~d}, J=8.5 \mathrm{~Hz}, 2 \mathrm{H}), 7.87$ $(\mathrm{d}, J=15.5 \mathrm{~Hz}, 1 \mathrm{H}), 7.70-7.67(\mathrm{~m}, 2 \mathrm{H}), 7.59(\mathrm{~d}, J=15.5 \mathrm{~Hz}, 1 \mathrm{H})$, 7.47-7.38 (m, 7H), 4.11-4.03 (m, 6H), 1.87-1.76 (m, 6H), 1.53$1.47(\mathrm{~m}, 6 \mathrm{H}), 1.38-1.26(\mathrm{~m}, 24 \mathrm{H}), 0.91-0.87(\mathrm{~m}, 9 \mathrm{H}) ;{ }^{13} \mathrm{C} \mathrm{NMR}$ $\left(125 \mathrm{MHz}, \mathrm{CDCl}_{3}\right)(\delta \mathrm{ppm}): 189.82,164.71,163.66,154.76$, 153.70, 153.34, 153.04, 151.57, 150.58, 150.34, 150.24, 147.02, $145.37,143.27,139.75,134.79,130.76,129.59,129.03,128.57$, 124.52, 123.42, 123.04, 122.54, 122.19, 121.95, 111.07, 108.64, $74.17,73.63,69.31,31.92,31.68,31.88,31.87,31.84,31.82$, $30.31,30.20,29.52,29.47,29.43,29.36,29.34,29.31,29.30$, 29.27, 26.09, 26.02, 26.01, 22.69, 14.12. HRMS, MALDI ${ }^{+} \mathrm{m} / \mathrm{z}$ : calcd for $\mathrm{C}_{52} \mathrm{H}_{68} \mathrm{~N}_{2} \mathrm{NaO}_{6}$ : $[\mathrm{M}+\mathrm{Na}]^{+}$839.4975, found: 839.4939.

IIc-14. Yield $53.6 \%$, mp $87-90{ }^{\circ} \mathrm{C}, R_{\mathrm{f}}=0.29\left(\mathrm{CH}_{2} \mathrm{Cl}_{2}\right.$ : petroleum ether : ethyl acetate $=3 \mathrm{~mL}: 1 \mathrm{~mL}: 20 \mu \mathrm{L})$; IR $(\mathrm{KBr}$, $\left.\nu, \mathrm{cm}^{-1}\right): 3063,2920,2850,1744,1658,1576,1495,1261,1011$, 879.80; ${ }^{1} \mathrm{H}$ NMR $\left(500 \mathrm{MHz}, \mathrm{CDCl}_{3}\right)(\delta \mathrm{ppm}): 8.19(\mathrm{~d}, J=8.5 \mathrm{~Hz}$, $2 \mathrm{H}), 8.06(\mathrm{~d}, J=8.5 \mathrm{~Hz}, 2 \mathrm{H}), 8.03(\mathrm{~d}, J=8.5 \mathrm{~Hz}, 2 \mathrm{H}), 7.87(\mathrm{~d}, J=$ $15.5 \mathrm{~Hz}, 1 \mathrm{H}), 7.70-7.68(\mathrm{~m}, 2 \mathrm{H}), 7.59(\mathrm{~d}, J=15.5 \mathrm{~Hz}, 1 \mathrm{H}), 7.46-$ $7.43(\mathrm{~m}, 5 \mathrm{H}), 7.38(\mathrm{~s}, 1 \mathrm{H}), 4.12-4.02(\mathrm{~m}, 6 \mathrm{H}), 1.87-1.75(\mathrm{~m}, 6 \mathrm{H})$, 1.53-1.45 (m, 6H), 1.40-1.26 (m, 60H), 0.91-0.86 (m, 9H); ${ }^{13} \mathrm{C}$ NMR $\left(125 \mathrm{MHz}, \mathrm{CDCl}_{3}\right)(\delta \mathrm{ppm}): 189.83,163.67,154.77,153.35$, 153.04, 151.58, 150.59, 150.35, 147.03, 145.38, 139.76, 134.80, $130.76,129.59,129.04,128.58,124.52,123.43,123.04,122.60$, $122.53,122.24,122.02,111.10,108.61,74.31,74.22,73.61$, $69.32,69.31,69.28,31.96,31.95,31.92,31.84,31.82,30.48$, $30.35,30.26,30.25,29.58,29.57,29.48,29.46,29.45,29.36$, 
$29.35,29.34,29.33,29.26,26.16,26.10,26.08,26.05,22.69$, 14.12. HRMS, MALDI ${ }^{+} \mathrm{m} / \mathrm{z}$ : calcd for $\mathrm{C}_{70} \mathrm{H}_{104} \mathrm{~N}_{2} \mathrm{NaO}_{6}$ : $[\mathrm{M}+\mathrm{Na}]^{+}$ 1091.7792, found: 1901.7763 .

IId-8. Yield 53.1\%, mp 90-91 ${ }^{\circ} \mathrm{C}, R_{\mathrm{f}}=0.2\left(\mathrm{CH}_{2} \mathrm{Cl}_{2}\right.$ : petroleum ether : ethyl acetate $=3 \mathrm{~mL}: 1 \mathrm{~mL}: 20 \mu \mathrm{L})$; IR $\left(\mathrm{KBr}, \nu, \mathrm{cm}^{-1}\right)$ : 3072, 2922, 2854, 1727, 1651, 1586, 1498, 1283, 1028, 942; ${ }^{1} \mathrm{H}$ NMR (500 MHz, $\left.\mathrm{CDCl}_{3}\right)(\delta \mathrm{ppm}): 8.17(\mathrm{~d}, J=8.5 \mathrm{~Hz}, 2 \mathrm{H}), 8.06(\mathrm{~d}$, $J=8.5 \mathrm{~Hz}, 2 \mathrm{H}), 8.02(\mathrm{~d}, J=8.5 \mathrm{~Hz}, 2 \mathrm{H}), 8.00(\mathrm{~d}, J=15.5 \mathrm{~Hz}, 1 \mathrm{H})$, $7.46(\mathrm{~d}, J=5.0 \mathrm{~Hz}, 1 \mathrm{H}, \mathrm{TpH}), 7.44-7.37(\mathrm{~m}, 6 \mathrm{H}), 7.13-7.11(\mathrm{~m}$, $1 \mathrm{H})$, 4.11-4.04 (m, 6H), 1.87-1.75 (m, 6H), 1.53-1.47 (m, 6H), 1.40-1.26 (m, 24H), 0.91-0.87 (m, 9H); ${ }^{13} \mathrm{C}$ NMR (125 MHz, $\left.\mathrm{CDCl}_{3}\right)(\delta \mathrm{ppm}): 189.11,164.71,163.66,154.77,153.70,153.34$, 153.04, 151.58, 150.59, 147.02, 143.27, 140.33, 139.65, 137.69, 132.41, 129.49, 129.13, 128.46, 124.52, 123.48, 123.04, 122.64, 122.53, 120.62, 111.08, 108.65, 74.31, 73.63, 69.32, 31.92, 31.83, $30.37,30.32,30.21,29.53,29.47,29.44,29.39,29.37,29.35$, 29.32, 29.30, 29.27, 26.10, 26.07, 26.03, 26.02, 22.72, 22.69, 14.12. HRMS, MALDI ${ }^{+} \mathrm{m} / z$ : calcd for $\mathrm{C}_{50} \mathrm{H}_{66} \mathrm{~N}_{2} \mathrm{NaO}_{6} \mathrm{~S}$ : $[\mathrm{M}+\mathrm{Na}]^{+}$ 845.4538, found: 845.4539.

III-8. Yield $71 \%, \mathrm{mp} 186-187^{\circ} \mathrm{C}, R_{\mathrm{f}}=0.2\left(\mathrm{CH}_{2} \mathrm{Cl}_{2}\right.$ : petroleum ether : ethyl acetate $=3 \mathrm{~mL}: 1 \mathrm{~mL}: 20 \mu \mathrm{L})$; IR $\left(\mathrm{KBr}, \nu, \mathrm{cm}^{-1}\right)$ : 3060, 2954, 2922, 2852, 1744, 1658, 1629, 1572, 1511, 1266, 1084, 979; ${ }^{1} \mathrm{H}$ NMR (500 MHz, $\left.\mathrm{CDCl}_{3}\right)(\delta \mathrm{ppm}): 8.24(\mathrm{~d}, J=$ $7.5 \mathrm{~Hz}, 2 \mathrm{H}), 8.17$ (d, $J=8.5 \mathrm{~Hz}, 2 \mathrm{H}), 8.07$ (d, $J=8.5 \mathrm{~Hz}, 2 \mathrm{H}), 8.03$ (d, $J=8.5 \mathrm{~Hz}, 2 \mathrm{H}), 7.83(\mathrm{~d}, J=15.5 \mathrm{~Hz}, 1 \mathrm{H}), 7.68(\mathrm{t}, J=7.5 \mathrm{~Hz}$, $1 \mathrm{H}), 7.63(\mathrm{~d}, J=8.5 \mathrm{~Hz}, 2 \mathrm{H}), 7.55$ (t, $J=7.5 \mathrm{~Hz}, 2 \mathrm{H}), 7.45$ (d, $J=$ $15.5 \mathrm{~Hz}, 1 \mathrm{H}), 7.42$ (d, $J=8.5 \mathrm{~Hz}, 2 \mathrm{H}), 6.95(\mathrm{~d}, J=8.5 \mathrm{~Hz}, 2 \mathrm{H})$, $4.02(\mathrm{t}, J=6.5 \mathrm{~Hz}, 2 \mathrm{H}), 1.85-1.78(\mathrm{~m}, 2 \mathrm{H}), 1.50-1.44(\mathrm{~m}, 2 \mathrm{H})$, 1.38-1.30 (m, 8H), $0.89(\mathrm{t}, J=6.8 \mathrm{~Hz}, 3 \mathrm{H}) ;{ }^{13} \mathrm{C}$ NMR $(125 \mathrm{MHz}$, $\left.\mathrm{CDCl}_{3}\right)(\delta \mathrm{ppm}): 189.87,164.83,161.53,154.64,153.52,150.31$, $145.37,140.15,133.90,130.40,130.29,129.48,129.21,128.70$, $127.28,124.51,122.97,122.54,119.45,114.99,68.25,31.82$, $29.35,29.25,29.17,26.03,22.68,14.12$. HRMS, MALDI ${ }^{+} m / z$ : calcd for $\mathrm{C}_{36} \mathrm{H}_{37} \mathrm{~N}_{2} \mathrm{O}_{4}:[\mathrm{M}+\mathrm{H}]^{+}$561.2753, found: 561.2728 .

III-14. Yield $70.6 \%, \mathrm{mp} 165-166{ }^{\circ} \mathrm{C}, R_{\mathrm{f}}=0.2\left(\mathrm{CH}_{2} \mathrm{Cl}_{2}-\right.$ : petroleum ether : ethyl acetate $=3 \mathrm{~mL}: 1 \mathrm{~mL}: 20 \mu \mathrm{L})$, IR $\left(\mathrm{KBr}, \nu, \mathrm{cm}^{-1}\right): 3042,2954,2922,2852,1744,1658,1629,1572$, 1511, 1266, 1084, 979.70; ${ }^{1} \mathrm{H}$ NMR (500 MHz, $\left.\mathrm{CDCl}_{3}\right)(\delta \mathrm{ppm})$ : $8.24(\mathrm{~d}, J=7.0 \mathrm{~Hz}, 2 \mathrm{H}), 8.17$ (d, $J=8.5 \mathrm{~Hz}, 2 \mathrm{H}), 8.07$ (d, $J=$ $8.5 \mathrm{~Hz}, 2 \mathrm{H}), 8.03(\mathrm{~d}, J=8.5 \mathrm{~Hz}, 2 \mathrm{H}), 7.83(\mathrm{~d}, J=15.5 \mathrm{~Hz}, 1 \mathrm{H})$, $7.68(\mathrm{t}, J=7.5 \mathrm{~Hz}, 1 \mathrm{H}), 7.62(\mathrm{~d}, J=8.5 \mathrm{~Hz}, 2 \mathrm{H}), 7.55(\mathrm{t}, J=7.5 \mathrm{~Hz}$, $2 \mathrm{H}), 7.46(\mathrm{~d}, J=15.5 \mathrm{~Hz}, 1 \mathrm{H}), 7.42(\mathrm{~d}, J=8.5 \mathrm{~Hz}, 2 \mathrm{H}), 6.94(\mathrm{~d}, J=$ $8.5 \mathrm{~Hz}, 2 \mathrm{H}), 4.01(\mathrm{t}, J=6.5 \mathrm{~Hz}, 2 \mathrm{H}), 1.84-1.78(\mathrm{~m}, 2 \mathrm{H}), 1.51-1.43$ (m, 2H), 1.36-1.26 (m, 20H), $0.88(\mathrm{t}, J=6.9 \mathrm{~Hz}, 3 \mathrm{H}) ;{ }^{13} \mathrm{C} \mathrm{NMR}$ $\left(125 \mathrm{MHz}, \mathrm{CDCl}_{3}\right)(\delta \mathrm{ppm}): 189.87,164.83,161.53,154.64$, $150.31,145.37,133.90,130.35,129.48,128.70,127.28,124.51$, 122.97, 122.54, 119.45, 114.99, 77.28, 77.03, 76.77, 68.25, 31.82, 29.45-29.03, 26.03, 22.68, 14.12. HRMS, MALDI ${ }^{+} \mathrm{m} / \mathrm{z}$ : calcd for $\mathrm{C}_{42} \mathrm{H}_{49} \mathrm{~N}_{2} \mathrm{NaO}_{4}:[\mathrm{M}+\mathrm{Na}]^{+}$667.3506, found: 667.3519.

IV-8. Yield $42.5 \%$, mp $172-177{ }^{\circ} \mathrm{C}, R_{\mathrm{f}}=0.17\left(\mathrm{CH}_{2} \mathrm{Cl}_{2}\right.$ : petroleum ether : ethyl acetate $=2 \mathrm{~mL}: 1 \mathrm{~mL}: 20 \mu \mathrm{L})$; IR $(\mathrm{KBr}$, $\left.\nu, \mathrm{cm}^{-1}\right):$ 3072, 2955, 2922, 2851, 1729, 1656, 1597, 1511, 1262, 1070, 985; ${ }^{1} \mathrm{H}$ NMR (500 MHz, $\left.\mathrm{CDCl}_{3}\right)(\delta \mathrm{ppm}): 8.17(\mathrm{~d}, J=$ $8.5 \mathrm{~Hz}, 4 \mathrm{H}), 8.06$ (d, $J=9.0 \mathrm{~Hz}, 2 \mathrm{H}), 8.02(\mathrm{~d}, J=8.5 \mathrm{~Hz}, 2 \mathrm{H}), 7.83$ $(\mathrm{d}, J=15.5 \mathrm{~Hz}, 1 \mathrm{H}), 7.63(\mathrm{~d}, J=8.5 \mathrm{~Hz}, 2 \mathrm{H}), 7.46(\mathrm{~d}, J=15.5 \mathrm{~Hz}$, $1 \mathrm{H}), 7.40(\mathrm{~d}, J=9.0 \mathrm{~Hz}, 2 \mathrm{H}), 6.99(\mathrm{~d}, J=9.0 \mathrm{~Hz}, 2 \mathrm{H}), 6.95(\mathrm{~d}, J=$ $8.5 \mathrm{~Hz}, 2 \mathrm{H}), 4.06(\mathrm{t}, J=6.5 \mathrm{~Hz}, 2 \mathrm{H}), 4.02(\mathrm{t}, J=6.5 \mathrm{~Hz}, 2 \mathrm{H})$,
1.86-1.78 (m, 4H), 1.50-1.44 (m, 4H), 1.39-1.26 (m, 16H), 0.91$0.88(\mathrm{~m}, 6 \mathrm{H}) .{ }^{13} \mathrm{C} \mathrm{NMR}\left(125 \mathrm{MHz}, \mathrm{CDCl}_{3}\right)(\delta \mathrm{ppm}): 189.85$, 163.78 , 161.52, 154.67, 153.74, 150.17, 145.34, 140.09, 132.41, $130.40,129.47,127.28,124.46,122.95,122.61,121.13,119.45$, 114.99, 114.41, 77.29, 77.03, 76.78, 68.32, 31.82, 29.22, 26.02, 22.68, 14.13. HRMS, MALDI ${ }^{+} m / z$ : calcd for $\mathrm{C}_{44} \mathrm{H}_{53} \mathrm{~N}_{2} \mathrm{O}_{5}:[\mathrm{M}+$ $\mathrm{H}]^{+}$689.3954, found: 689.3922 .

IV-10. Yield $42.7 \%$, mp $149-150{ }^{\circ} \mathrm{C}, R_{\mathrm{f}}=0.18\left(\mathrm{CH}_{2} \mathrm{Cl}_{2}\right.$ : petroleum ether : ethyl acetate $=2 \mathrm{~mL}: 1 \mathrm{~mL}: 20 \mu \mathrm{L})$; IR $(\mathrm{KBr}$, $\left.\nu, \mathrm{cm}^{-1}\right): 3064,2955,2920,2851,1737,1658,1628,1573,1510$, 1258, 1080, 989; ${ }^{1} \mathrm{H} \mathrm{NMR}\left(500 \mathrm{MHz}, \mathrm{CDCl}_{3}\right)(\delta \mathrm{ppm}): 8.17$ (d, $J=$ $8.0 \mathrm{~Hz}, 4 \mathrm{H}), 8.06$ (d, $J=9.0 \mathrm{~Hz}, 2 \mathrm{H}), 8.02(\mathrm{~d}, J=8.5 \mathrm{~Hz}, 2 \mathrm{H}), 7.83$ $(\mathrm{d}, J=15.5 \mathrm{~Hz}, 1 \mathrm{H}), 7.63(\mathrm{~d}, J=8.5 \mathrm{~Hz}, 2 \mathrm{H}), 7.46(\mathrm{~d}, J=15.5 \mathrm{~Hz}$, $1 \mathrm{H}), 7.40$ (d, $J=8.5 \mathrm{~Hz}, 2 \mathrm{H}), 6.99$ (d, $J=9.0 \mathrm{~Hz}, 2 \mathrm{H}), 6.95$ (d, $J=$ $8.5 \mathrm{~Hz}, 2 \mathrm{H}), 4.06(\mathrm{t}, J=6.5 \mathrm{~Hz}, 2 \mathrm{H}), 4.01(\mathrm{t}, J=6.5 \mathrm{~Hz}, 2 \mathrm{H}), 1.86-$ $1.78(\mathrm{~m}, 4 \mathrm{H}), 1.51-1.44(\mathrm{~m}, 4 \mathrm{H}), 1.36-1.28$ (m, 24H), 0.90-0.87 $(\mathrm{m}, 6 \mathrm{H}) ;{ }^{13} \mathrm{C}$ NMR $\left(125 \mathrm{MHz}, \mathrm{CDCl}_{3}\right)(\delta \mathrm{ppm}):$ 189.86, 163.78, 161.52 , 154.67, 153.74, 145.34, 132.41, 130.40, 129.47, 127.28, $124.46,122.95,122.61,121.12,119.46,114.99,114.41,77.28$, 77.03, 76.78, 68.32, 31.91, 29.57, 29.36, 29.14, 26.01, 22.70, 14.14. HRMS, MALDI ${ }^{+} m / z$ : calcd for $\mathrm{C}_{48} \mathrm{H}_{61} \mathrm{~N}_{2} \mathrm{O}_{5}:[\mathrm{M}+\mathrm{H}]^{+}$ 745.4580, found: 745.4545 .

IV-12. Yield $43 \%, \mathrm{mp} 147-148{ }^{\circ} \mathrm{C}, R_{\mathrm{f}}=0.20\left(\mathrm{CH}_{2} \mathrm{Cl}_{2}\right.$ : petroleum ether : ethyl acetate $=2 \mathrm{~mL}: 1 \mathrm{~mL}: 20 \mu \mathrm{L})$; IR $(\mathrm{KBr}$, $\left.\nu, \mathrm{cm}^{-1}\right)$ : 3068, 2955, 2919, 2850, 1737, 1659, 1603, 1573, 1510, 1260, 1081, 989. ${ }^{1} \mathrm{H}$ NMR (500 $\left.\mathrm{MHz}, \mathrm{CDCl}_{3}\right)(\delta \mathrm{ppm}): 8.17(\mathrm{~d}, J=$ $8.0 \mathrm{~Hz}, 4 \mathrm{H}), 8.05(\mathrm{~d}, J=9.0 \mathrm{~Hz}, 2 \mathrm{H}), 8.02(\mathrm{~d}, J=8.5 \mathrm{~Hz}, 2 \mathrm{H}), 7.83$ $(\mathrm{d}, J=15.5 \mathrm{~Hz}, 1 \mathrm{H}), 7.63(\mathrm{~d}, J=8.5 \mathrm{~Hz}, 2 \mathrm{H}), 7.46(\mathrm{~d}, J=15.5 \mathrm{~Hz}$, $1 \mathrm{H}), 7.40(\mathrm{~d}, J=8.5 \mathrm{~Hz}, 2 \mathrm{H}), 6.99(\mathrm{~d}, J=9.0 \mathrm{~Hz}, 2 \mathrm{H}), 6.94(\mathrm{~d}, J=$ $8.5 \mathrm{~Hz}, 2 \mathrm{H}), 4.06(\mathrm{t}, J=6.5 \mathrm{~Hz}, 2 \mathrm{H}), 4.01(\mathrm{t}, J=6.5 \mathrm{~Hz}, 2 \mathrm{H}), 1.86-$ $1.78(\mathrm{~m}, 4 \mathrm{H}), 1.51-1.43(\mathrm{~m}, 4 \mathrm{H}), 1.37-1.27(\mathrm{~m}, 32 \mathrm{H}), 0.89(\mathrm{t}, J=$ $6.5 \mathrm{~Hz}, 6 \mathrm{H}) ;{ }^{13} \mathrm{C}$ NMR $\left(125 \mathrm{MHz}, \mathrm{CDCl}_{3}\right)(\delta \mathrm{ppm}): 189.85,164.57$, $163.78,161.52$, 154.67, 153.74, 150.17, 145.34, 140.09, 132.41, $130.40,129.47,127.28,124.46,122.95,122.61,121.12,119.45$, 114.98, 114.41, 77.29, 77.03, 76.78, 68.32, 31.94, 29.63, 29.37, 29.14, 26.01, 22.71, 14.15. HRMS, MALDI ${ }^{+} \mathrm{m} / \mathrm{z}$ : calcd for $\mathrm{C}_{52} \mathrm{H}_{69} \mathrm{~N}_{2} \mathrm{O}_{5}:[\mathrm{M}+\mathrm{H}]^{+}$801.5206, found: 801.5189.

IV-14. Yield $42.6 \%, \mathrm{mp} 140-144{ }^{\circ} \mathrm{C}, R_{\mathrm{f}}=0.23\left(\mathrm{CH}_{2} \mathrm{Cl}_{2}\right.$ : petroleum ether : ethyl acetate $=2 \mathrm{~mL}: 1 \mathrm{~mL}: 20 \mu \mathrm{L})$; IR $(\mathrm{KBr}$, $\left.\nu, \mathrm{cm}^{-1}\right)$ : 3062, 2955, 2918, 2849, 1736, 1659, 1603, 1568, 1258, 1082.04, 978. ${ }^{1} \mathrm{H}$ NMR (500 $\left.\mathrm{MHz}, \mathrm{CDCl}_{3}\right)$ trans isomer $>85 \%(\delta$ ppm): 8.17 (d, $J=8.0 \mathrm{~Hz}, 4 \mathrm{H}), 8.05(\mathrm{~d}, J=8.5 \mathrm{~Hz}, 2 \mathrm{H}), 8.01(\mathrm{~d}, J$ $=8.5 \mathrm{~Hz}, 2 \mathrm{H}), 7.82(\mathrm{~d}, J=16.0 \mathrm{~Hz}, 1 \mathrm{H}), 7.63(\mathrm{~d}, J=8.5 \mathrm{~Hz}, 2 \mathrm{H})$, $7.46(\mathrm{~d}, J=15.5 \mathrm{~Hz}, 1 \mathrm{H}), 7.40(\mathrm{~d}, J=9.0 \mathrm{~Hz}, 2 \mathrm{H}), 6.99$ (d, $J=$ $9.0 \mathrm{~Hz}, 2 \mathrm{H}), 6.94(\mathrm{~d}, J=8.5 \mathrm{~Hz}, 2 \mathrm{H}), 4.06(\mathrm{t}, J=6.5 \mathrm{~Hz}, 2 \mathrm{H}), 4.01$ $(\mathrm{t}, J=6.5 \mathrm{~Hz}, 2 \mathrm{H}), 1.86-1.78(\mathrm{~m}, 4 \mathrm{H}), 1.51-1.44(\mathrm{~m}, 4 \mathrm{H}), 1.37-$ $1.24(\mathrm{~m}, 40 \mathrm{H}), 0.88(\mathrm{t}, J=6.5 \mathrm{~Hz}, 6 \mathrm{H}) .{ }^{13} \mathrm{C}$ NMR $(125 \mathrm{MHz}$, $\left.\mathrm{CDCl}_{3}\right)(\delta \mathrm{ppm}): 189.84,164.52(\mathrm{~s}), 163.79,161.57,154.57$, 153.74 , 150.17, 145.32, 140.08, 132.38, 130.42, 129.48, 127.31, 124.42, 122.93, 122.59, 121.12, 119.44, 114.95, 114.46, 77.30, 77.04, 76.77, 68.31, 31.96, 29.64, 29.36, 29.14, 26.03, 22.71, 14.14. HRMS, MALDI ${ }^{+} \mathrm{m} / z$ : calcd for $\mathrm{C}_{56} \mathrm{H}_{77} \mathrm{~N}_{2} \mathrm{O}_{5}:[\mathrm{M}+\mathrm{H}]^{+}$ 857.5832, found: 857.5812 .

V. Yield $42.7 \%, \mathrm{mp} 220-224{ }^{\circ} \mathrm{C}, R_{\mathrm{f}}=0.18\left(\mathrm{CH}_{2} \mathrm{Cl}_{2}\right.$ : petroleum ether : ethyl acetate $=2 \mathrm{~mL}: 1 \mathrm{~mL}: 20 \mu \mathrm{L})$; IR $\left(\mathrm{KBr}, \nu, \mathrm{cm}^{-1}\right)$ : 3070, 2923, 2852, 1731, 1655, 1598, 1570, 1510, 1246, 1064, 979; ${ }^{1} \mathrm{H}$ NMR $\left(500 \mathrm{MHz}, \mathrm{CDCl}_{3}\right)$ trans isomer $>70 \%(\delta \mathrm{ppm}): 8.76$ 
(s, 1H), $8.33(\mathrm{~d}, J=9.0 \mathrm{~Hz}, 1 \mathrm{H}), 8.27$ (d, $J=9.0 \mathrm{~Hz}, 1 \mathrm{H}), 8.18(\mathrm{~d}, J$ $=8.5 \mathrm{~Hz}, 3 \mathrm{H}), 8.09(\mathrm{~d}, J=8.5 \mathrm{~Hz}, 2 \mathrm{H}), 8.03(\mathrm{~d}, J=8.5 \mathrm{~Hz}, 2 \mathrm{H})$, $7.94(\mathrm{~d}, J=9.0 \mathrm{~Hz}, 1 \mathrm{H}), 7.84(\mathrm{~d}, J=15.5 \mathrm{~Hz}, 1 \mathrm{H}), 7.63(\mathrm{~d}, J=$ $8.5 \mathrm{~Hz}, 2 \mathrm{H}), 7.49-7.44(\mathrm{~m}, 3 \mathrm{H}), 7.39$ (d, $J=9.0 \mathrm{~Hz}, 1 \mathrm{H}), 7.26(\mathrm{~s}$, $1 \mathrm{H}), 6.95(\mathrm{~d}, J=8.5 \mathrm{~Hz}, 2 \mathrm{H}), 4.24(\mathrm{t}, J=6.5 \mathrm{~Hz}, 2 \mathrm{H}), 4.02(\mathrm{t}, J=$ $6.5 \mathrm{~Hz}, 2 \mathrm{H}), 1.95-1.87(\mathrm{~m}, 2 \mathrm{H}), 1.84-1.78(\mathrm{~m}, 2 \mathrm{H}), 1.58-1.53(\mathrm{~m}$, $2 \mathrm{H}), 1.49-1.45(\mathrm{~m}, 2 \mathrm{H}), 1.42-1.27(\mathrm{~m}, 16 \mathrm{H}), 0.90(\mathrm{t}, J=6.5 \mathrm{~Hz}$, $6 \mathrm{H}) ;{ }^{13} \mathrm{C} \mathrm{NMR}\left(125 \mathrm{MHz}, \mathrm{CDCl}_{3}\right)(\delta \mathrm{ppm}): 189.84,164.79,161.53$, 154.60, 145.36, 140.15, 132.32, 130.40, 129.56, 127.28, 126.84, 124.62, 124.21, 122.98, 122.58, 119.43, 115.58, 114.99, 77.29, $77.03,76.78,70.03,68.25,31.83,29.28,25.99,22.68,14.13$. HRMS, MALDI ${ }^{+} m / z$ : calcd for $\mathrm{C}_{48} \mathrm{H}_{55} \mathrm{~N}_{2} \mathrm{O}_{5}$ : $[\mathrm{M}+\mathrm{H}]^{+}$739.4111, found: 739.4108 .

\section{Conflicts of interest}

There are no conflicts to declare.

\section{Acknowledgements}

We thank the National Natural Science Foundation of China (NSFC, 21562032), Natural Science Foundation of Inner Mongolia (2017MS0205) and Research Program of Science and Technology at Universities of Inner Mongolia (NJZZ001) for their generous financial support.

\section{References}

1 S. Laschat, A. Baro, N. Steinke, F. Giesselmann, C. Hägele, G. Scalia, R. Judele, E. Kapatsina, S. Sauer, A. Schreivogel and M. Tosoni, Angew. Chem., 2007, 119, 4916.

2 G. Lee, R. J. Carlton, F. Araoka, N. L. Abbott and H. Takezoe, Adv. Mater., 2013, 25, 245.

3 A. M. Lowe and N. L. Abbott, Chem. Mater., 2012, 24, 746.

4 A. D. Price and D. K. Schwartz, J. Am. Chem. Soc., 2008, 130, 8188.

5 S. Dixit and R. A. Vora, Mol. Cryst. Liq. Cryst., 2015, 623, 56. 6 Z. Jiang, M. Xu, F. Li and Y. Yu, J. Am. Chem. Soc., 2013, 135, 16446.

7 G. Hegde, G. Shanker, S. M. Gan, A. R. Yuvaraj, S. Mahmood and U. K. Mandal, Liq. Cryst., 2016, 43, 1578.

8 J. Wang, Y. Shi, K. Yang, J. Wei and J. Guo, $R S C A d v .$, 2015, 5, 67357.

9 M. L. Rahman, T. K. Biswas, S. M. Sarkar, M. M. Yusoff, M. N. F. A. Malek and C. Tschierske, J. Mol. Liq., 2015, 202, 125.

10 I. Niezgoda, J. Jaworska and Z. Galewski, J. Mol. Liq., 2016, 222, 571 .

11 N. G. Nagaveni, M. Gupta, A. Royb and V. Prasad, J. Mater. Chem., 2010, 20, 9089.

12 N. Trišović, J. Antanasijević, T. Tóth-Katona, M. Kohout, M. Salamonczyk, S. Sprunt, A. Jákli and K. Fodor-Csorba, RSC Adv., 2015, 5, 64886.

13 N. G. Nagaveni, A. Royb and V. Prasad, J. Mater. Chem., 2012, 22, 8948.
14 S. H. Ryu, M. Gim, W. Lee, S. W. Choi and D. K. Yoon, ACS Appl. Mater. Interfaces, 2017, 9, 3186.

15 D. Y. Kim, S. A. Lee, H. Kim, S. M. Kim, N. Kimc and K. U. Jeong, Chem. Commun., 2015, 51, 11080.

16 A. Zep, K. Sitkowska, D. Pociecha and E. Gorecka, J. Mater. Chem. C, 2014, 2, 2323.

17 H. Y. Zhong, L. Chen, R. Yang, Z. Y. Meng, X. M. Ding, X. F. Liu and Y. Z. Wang, J. Mater. Chem. C, 2017, 5, 3306.

18 E. Verploegen, J. Soulages, M. Kozberg, T. Zhang, G. McKinley and P. Hammond, Angew. Chem., Int. Ed., 2009, 48, 3494.

19 J. Wang, C. Lin, J. Zhang, J. Wei, Y. Song and J. Guo, J. Mater. Chem. C, 2015, 3, 4179.

20 R. Prasath, P. Bhavana, S. W. Ng and E. R. T. Tiekink, J. Organomet. Chem., 2013, 726, 62.

21 S. Attar, Z. O'Brien, H. Alhaddad, M. L. Golden and A. Calderón-Urrea, Bioorg. Med. Chem., 2011, 19, 2055.

22 C. A. Calliste, J. C. Le Bail, P. Trouilas, C. Poug, A. J. Chulia and L. J. Doroux, Anticancer Res., 2001, 21, 3949.

23 X. Wu, E. R. T. Tiekink, I. Kostetski, N. Kocherginsky, A. L. C. Tan, S. B. Khoo, P. Wilairat and M. L. Go, Eur. J. Pharm. Sci., 2006, 27, 175.

24 P. S. Patil, S. M. Dharmaprakash, K. Ramakrishna and H. K. Fun, J. Cryst. Growth, 2007, 303, 520.

25 H. Zhao, X. Zhu, D. Wang, S. Chen and Z. Bian, Aust. J. Chem., 2015, 68, 1035.

26 R. L. Coelho, E. Westphal, D. Z. Mezalira and H. Gallardo, Liq. Cryst., 2017, 44, 405.

27 H. T. Srinivasa and S. Kumar, Liq. Cryst., 2017, 44, 1506.

28 A. Karuppusamy, V. Ramkumar, P. Kannan, S. Balamurugan and S. M. Said, Soft Mater., 2017, 15, 132.

29 B. B. Jain and R. B. Patel, Mol. Cryst. Liq. Cryst., 2016, 638, 27.

30 N. R. Muniya and V. R. Patel, Mol. Cryst. Liq. Cryst., 2016, 638, 95.

31 A. S. Pandey, R. Dhar, A. S. Achalkumar and C. V. Yelamaggad, Liq. Cryst., 2011, 38, 775.

32 J. Li, W. Zhang, Y. Zhao and J. Pu, Adv. Mater. Res., 2012, 380, 326.

33 D. Jayalatha, R. Balamurugan and P. Kannan, High Perform. Polym., 2009, 21, 139.

34 H. Zhao, X. Zhu, Y. Shang, S. Chen, B. Li and Z. Bian, RSC Adv., 2016, 6, 34476.

35 C. Selvarasu and P. Kannan, J. Mol. Struct., 2016, 1125, 234. 36 T. Ogata, K. Oikawa, T. Fujisawa, S. Motoyama, T. Izumi, A. Kasahara and N. Tanaka, Bull. Chem. Soc. Jpn., 1981, 54, 3723. 37 S. Y. Kim, O. N. Kadkin, E. H. Kim and M. G. Choi, J. Organomet. Chem., 2011, 696, 2429.

38 G. Casella, V. Causin, F. Rastrelli and G. Saielli, Liq. Cryst., 2016, 43, 1161.

39 I. Cârlescu, A. M. Scutar, D. Apreutesei, V. Alupei and D. Scutaru, Liq. Cryst., 2007, 34, 775. 